\title{
Higgs pair production from bottom quark annihilation to NNLO in QCD
}

\author{
A.H. Ajjath, ${ }^{a}$ Pulak Banerjee, ${ }^{a}$ Amlan Chakraborty, ${ }^{a}$ Prasanna K. Dhani, ${ }^{a, b}$ \\ Pooja Mukherjee, ${ }^{a}$ Narayan Rana ${ }^{c, d}$ and V. Ravindran ${ }^{a}$ \\ ${ }^{a}$ The Institute of Mathematical Sciences, HBNI, \\ Taramani, Chennai 600113, India \\ ${ }^{b}$ INFN - Sezione di Firenze, \\ I-50019 Sesto Fiorentino, Florence, Italy \\ ${ }^{c}$ Deutsches Elektronen-Synchrotron, DESY, \\ Platanenallee 6, D-15738 Zeuthen, Germany \\ ${ }^{d}$ INFN - Sezione di Milano, \\ Via Celoria 16, I-20133 Milano, Italy \\ E-mail: ajjathah@imsc.res.in, bpulak@imsc.res.in, \\ amlanchak@imsc.res.in, prasannakumar.dhani@fi.infn.it, \\ poojamukherjee@imsc.res.in, narayan.rana@mi.infn.it, \\ ravindra@imsc.res.in
}

ABSTRACT: We present the first results on the two-loop massless QCD corrections to the four-point amplitude $b+\bar{b} \rightarrow H+H$ in the five flavor scheme, treating bottom quarks as massless. This amplitude is sensitive to the trilinear Higgs boson coupling. Our two-loop result for this amplitude constitutes of purely virtual contributions to the next-to-next-toleading order QCD predictions for the production of a pair of Higgs bosons at the Large Hadron Collider. Using these two loop amplitudes and exploiting the universality of the soft contributions in perturbative QCD, we obtain the NNLO QCD effects in the soft plus virtual approximation. We find that the inclusion of higher order terms reduce the uncertainties resulting from the unphysical renormalisation and factorisation scales.

KEYwords: NLO Computations, QCD Phenomenology

ARXiv EPrint: 1811.01853 


\section{Contents}

1 Introduction 1

2 Theory 3

2.1 The Yukawa interaction 4

2.2 Kinematics 5

2.3 General structure of the amplitude 5

2.4 Classification of Feynman diagrams 6

3 Methodology $\quad 7$

$\begin{array}{lll}3.1 & \text { Computational details } & 7\end{array}$

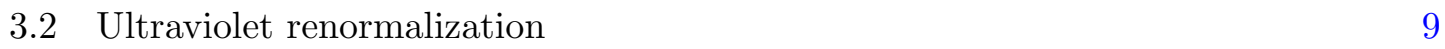

$\begin{array}{lll}3.3 & \text { Infrared divergences and their factorization } & 10\end{array}$

4 Numerical evaluation of the amplitude $\quad 12$

5 Inclusive cross section up to NNLO 13

$\begin{array}{llr}6 & \text { Phenomenology } & 20\end{array}$

$\begin{array}{llr}7 & \text { Conclusion } & 23\end{array}$

\section{Introduction}

Ever since the discovery of the Standard Model (SM) Higgs boson [1, 2], one of the main objectives of the Large Hadron Collider (LHC) physics program has been to understand its properties. This involves the measurements of the Higgs boson couplings to the SM fermions and gauge bosons, its mass $\left(m_{h}\right)$, its CP properties etc. Among these, the Higgs boson self couplings such as the trilinear $\left(\lambda_{3}^{\mathrm{SM}}\right)$ and quartic couplings $\left(\lambda_{4}^{\mathrm{SM}}\right)$ take prominence, which in the SM, can be unambiguously obtained from the Higgs boson mass. The SM Higgs potential, after the electro-weak symmetry breaking (EWSB), is given by

$$
\mathcal{L} \supset-\frac{m_{h}^{2}}{2} \phi^{2}(x)-\lambda_{3}^{\mathrm{SM}} v \phi^{3}(x)-\lambda_{4}^{\mathrm{SM}} \phi^{4}(x), \quad \lambda_{3}^{\mathrm{SM}}=\frac{m_{h}^{2}}{2 v^{2}}, \quad \lambda_{4}^{\mathrm{SM}}=\frac{m_{h}^{2}}{8 v^{2}},
$$

where $\phi(x)$ denotes the Higgs field. $v \approx 246 \mathrm{GeV}$ is the vacuum expectation value (vev) of the Higgs field and is fixed by the Fermi constant $G_{F}$. The Higgs boson mass $m_{h}$, is found experimentally to be approximately equal to $125 \mathrm{GeV}$ and hence, the SM values for $\lambda_{3}^{\mathrm{SM}}$ and $\lambda_{4}^{\mathrm{SM}}$ are $\sim 0.13$ and $\sim 0.03$, respectively. However, presence of beyond the SM (BSM) physics scenarios can modify these couplings, which, in turn, suggests independent measurements of them. Any deviation from the SM values from the experimental 
measurements, could provide crucial information on the structure of the scalar potential and thus could constrain BSM physics scenarios [3]. Moreover, the measurement of $\lambda_{3}^{\mathrm{SM}}$ also provides a way to check that the EWSB follows from the simple Ginzburg-Landau $\phi^{4}$ potential. The observable that can probe these couplings at the hadron colliders is the production of multiple Higgs bosons [4]. More precisely, the production of a pair of Higgs bosons can probe $\lambda_{3}^{\mathrm{SM}}$ but it is difficult to measure due to the smallness of its production cross section and the presence of a large QCD background. However, the study for the high luminosity LHC indicate that the Higgs boson pair production due to gluon fusion can predict $\lambda_{3}^{\mathrm{SM}}$ with $\mathcal{O}(1)$ accuracy [5-8].

A pair of Higgs bosons can be produced through several partonic channels, viz gluon fusion, vector boson fusion, associated production with a vector boson or a pair of heavy quarks. Among these channels, the gluon fusion channel is the most dominant one at the LHC. Being a loop-induced channel, gluon fusion gives a minuscule production cross section. Additionally, the large background of this channel makes its measurement experimentally challenging. Hence unless contributions from BSM physics enhance the production cross section, a measurement of this channel will require a considerable integrated luminosity. On the other hand, in such a scenario, the sub-dominant channels in the SM could possibly become interesting as they would receive substantial contributions from new physics. One such channel is the production of a pair of Higgs bosons in bottom quark annihilation. In certain supersymmetric models, namely the Minimal Supersymmetric SM (MSSM) [9], the bottom quark Yukawa coupling is enhanced w.r.t. the top quark Yukawa coupling, in the large $\tan \beta$ region, where $\tan \beta$ is the ratio of vev's of up and down type Higgs fields in the Higgs sector of the MSSM. Hence precise predictions for this channel is of high importance.

The dominant channel for Higgs boson pair production i.e. the gluon fusion channel, is mediated by a top quark loop. This was evaluated at leading order (LO) in perturbative QCD in [10-12] decades before. The next-to-leading order (NLO) contributions were obtained in [13] only in the infinite top mass limit, i.e. the top quark loop is integrated out resulting in an effective Lagrangian [13-16] of gluons and Higgs fields. There are several NLO results [17-22] considering finite top quark mass effects which finally led to the full NLO corrections with exact top quark mass dependence [23, 24]. In all these works, it has been found that, with an inclusive $K$-factor close to 2, the QCD corrections at NLO level are as large as that observed for a single Higgs boson production. Hence, the next-to-nextto-leading order (NNLO) corrections were computed in [25], in the effective theory, followed by a soft plus virtual (SV) approximated NNLO cross section in [26]. Consecutively, the effect of leading top quark mass corrections also has been included in [27]. Finally a fully differential distribution has been obtained at the NNLO level in $[28,29]$ and also threshold resummation at next-to-next-to-leading logarithmic (NNLL) level in [30, 31]. In [32], a reweighting technique has been used to properly account for finite top mass effects at NNLO level. Recently the virtual contributions relevant for next-to-next-to-next-to-leading order $\left(\mathrm{N}^{3} \mathrm{LO}\right)$ QCD have also been computed in [33], within the effective theory.

While a plethora of work has been performed to reach ultimate precision for the gluon channel, the sub-dominant channels have not received much attention. Although, as mentioned earlier, in certain BSM physics scenarios, they become consequential. We are partic- 
ularly interested in the bottom quark annihilation channel where the Higgs boson couples to bottom quarks through the Yukawa coupling (proportional to the mass of the bottom quark), and the bottom quark is massless otherwise [34-36]. For single Higgs boson production through this channel, various work is known up to NNLO [37-42] and $\mathrm{N}^{3} \mathrm{LO}$ [43-46] level in the variable flavor scheme (VFS) [47-50]. For the production of a pair of Higgs bosons, the NLO correction was first obtained in [51]. Later on, NLO corrections have been obtained for this channel considering several BSM scenarios [52-54]. For the latter, the bottom quark annihilation process dominates over the gluon fusion even at LO level. In addition, their NLO QCD corrections are not only sizeable but also larger than the supersymmetric QCD corrections. In order to stabilize the cross section with respect to higher order radiative corrections, NNLO corrections to this channel are desirable. In this paper, as a first step, we present full NNLO QCD corrections from certain class of diagrams to inclusive cross section for producing pair of Higgs bosons at the LHC and apply soft plus virtual approximation for the other class of diagrams. We find that the latter is sub-dominant and hence this approximation is good enough for phenomenological studies at the LHC.

There are two classes of diagrams (we call them Class-A and Class-B, see section 2.4), that contribute at two loops. The vertex type of diagrams which belong to Class-A are already known up to three loops [44]. For the Class-B, the one-loop QCD corrections exist in the literature [51]. Here we compute the two-loop QCD corrections. We have studied the structure of infrared (IR) singularities and found that they are in agreement with the predictions by Catani [55]. The finite results expressed in terms of classical polylogarithms of weight up to 4 is used to study the numerical stability of the amplitudes over a wide range of allowed kinematical variables. These amplitudes constitute important component of NNLO predictions for the observables related to the production of a pair of Higgs bosons at the LHC. In general, these amplitudes suitably combined with the universal soft gluon contributions from the real emission diagrams can be used to obtain soft plus virtual contributions up to NNLO level. We follow this approach [56] for the class B diagrams, while for the class A diagrams we can suitably use results that are already available for the single production up to NNLO level.

The paper is organized as follows. In section 2, we discuss the Lagrangian, kinematics and the classes of diagrams that are relevant for our computation. Section 3 contains details of the computation, the ultraviolet (UV) renormalization and the structure of IR divergences. We devote section 4 for the numerical evaluation of the amplitude over a wide kinematic region. In section 5, we present relevant analytic results that are required to compute inclusive cross section for producing pair of Higgs bosons using the amplitudes computed up to two-loop level. In section 6 , we study their numerical impact at the LHC. Finally, we conclude in section 7 .

\section{Theory}

At the LHC, the dominant channel for the production of a pair of Higgs bosons is the gluon fusion. In addition, there are several sub-leading channels that contribute to the 
production. We consider one of these channels, namely the production through the bottom quark annihilation process. Since the LO and the NLO [51] QCD effects have already been studied in the literature, as a first step towards the computation of the full NNLO QCD corrections, we evaluate two-loop virtual contributions to the production of a pair of Higgs bosons in this channel. Note that we further need to compute contributions from real emission sub-processes to obtain IR safe observables at the NNLO level. These pure virtual corrections contribute to both the inclusive as well as the differential observables. These results along with the process independent soft gluon contributions, can give us the first result at the NNLO level in the threshold limit, i.e., when the invariant mass of the pair of Higgs bosons approaches the partonic center of mass energy.

We use the regularized version of the QCD Lagrangian throughout. The regularization scheme that we use, is the dimensional regularization (DR), in which all the fields and couplings of the Lagrangian and the loop integrals that appear in the Feynman diagrams are analytically continued to $d=4+\epsilon$ space-time dimensions. In addition, we perform traces of Dirac $\gamma$ matrices in $d$-dimensions.

\subsection{The Yukawa interaction}

We begin by reviewing the theoretical framework for the production of a pair of Higgs bosons via bottom quark annihilation at hadron colliders. The interaction part of the Lagrangian that is responsible for the production is given by,

$$
\mathcal{L}=-\lambda_{b} \phi(x) \bar{\psi}_{b}(x) \psi_{b}(x)
$$

where $\psi_{b}(x)$ is the bottom quark field. $\lambda_{b}$ is the Yukawa coupling which after the EWSB is found to be $m_{b} / v$, where $m_{b}$ is the bottom quark mass and $v$ the vev of the Higgs field. In the SM, the ratio of the top quark Yukawa coupling $\left(\lambda_{t}\right)$ and the bottom quark Yukawa coupling $\left(\lambda_{b}\right)$ is found to be approximately 35 i.e. $\lambda_{t} / \lambda_{b} \approx 35$. In addition, the bottom quark flux in the proton-proton collision is much smaller than the gluon flux. Hence, the contribution from this channel is sub-dominant as compared to the gluon fusion channel. However, in the MSSM [9], this ratio depends on the value of $\tan \beta$ which can increase the contribution resulting from the bottom quark annihilation channel. At LO,

$$
\frac{\lambda_{t}^{\mathrm{MSSM}}}{\lambda_{b}^{\mathrm{MSSM}}}=f_{\phi}(\alpha) \frac{m_{t}}{m_{b}} \frac{1}{\tan \beta},
$$

with

$$
f_{\phi}(\alpha)=\left\{\begin{array}{r}
-\cot \alpha \text { for } \phi=h, \\
\tan \alpha \text { for } \phi=H, \\
\cot \beta \text { for } \phi=A,
\end{array}\right.
$$

where $h$ is the SM like light Higgs boson, $H$ and $A$ are the heavy and the pseudoscalar Higgs bosons, respectively. The parameter $\alpha$ is the angle between weak and mass eigenstates of the neutral Higgs bosons $h$ and $H$. Since, the bottom quark mass is much smaller than the other energy scales that appear at the partonic level, we set the former to zero except in the Yukawa coupling in perturbation theory [34-36]. In particular, the finite mass effects 
from the bottom quarks are found to be suppressed by the inverse power of mass of the Higgs boson. The number of active flavors is taken to be $n_{f}=5$ and we work in the Feynman gauge.

\subsection{Kinematics}

We compute all the relevant one- and two-loop amplitudes in perturbative QCD that contribute to the annihilation of bottom quarks into a pair of Higgs bosons. The scattering process is given by

$$
b\left(p_{1}\right)+\bar{b}\left(p_{2}\right) \rightarrow H\left(p_{3}\right)+H\left(p_{4}\right),
$$

where $p_{1}, p_{2}$ are the momenta of incoming bottom, anti-bottom quarks and $p_{3}, p_{4}$ are the momenta of the final state Higgs bosons. The associated Mandelstam variables are,

$$
s=\left(p_{1}+p_{2}\right)^{2}, \quad t=\left(p_{1}-p_{3}\right)^{2}, \quad u=\left(p_{2}-p_{3}\right)^{2},
$$

which satisfy the relation $s+t+u=2 m_{h}^{2}$. For convenience, we use the dimensionless variables $x, y$ and $z$ defined [57] as follows

$$
s=m_{h}^{2} \frac{(1+x)^{2}}{x}, \quad t=-m_{h}^{2} y, \quad u=-m_{h}^{2} z .
$$

The variables $x, y$ and $z$ satisfy

$$
\frac{(1+x)^{2}}{x}-y-z=2
$$

The final result will be expressed in term of logarithms and classical polylogarithms which are functions of these scaling variables.

\subsection{General structure of the amplitude}

The external states for the process given in eq. (2.4) involve two fermions and two scalars, hence the most general structure of the amplitude can be parameterized as

$$
\begin{aligned}
\mathcal{A}_{i j} & =\bar{v}\left(p_{2}\right)\left(\mathcal{C}_{1}+\mathcal{C}_{2} \not p_{3}\right) u\left(p_{1}\right) \delta_{i j} \\
& \equiv\left(\mathcal{C}_{1} \mathcal{T}_{1}+\mathcal{C}_{2} \mathcal{T}_{2}\right) \delta_{i j}
\end{aligned}
$$

where the coefficients $\mathcal{C}_{m} \equiv \mathcal{C}_{m}(x, y, z)$ with $m=1,2$ are scalar functions. In color space, the amplitude is diagonal in the indices $(i, j)$ of the incoming quarks. Since, we are interested in higher order QCD corrections, we have used symmetries such as Lorentz covariance, parity and time reversal invariances to parameterize the amplitude. In addition we have dropped those terms that vanish when the bottom quarks are massless. The coefficients $\mathcal{C}_{m}, m=1,2$, can be determined from the amplitude $\mathcal{A}_{i j}$ by using appropriate projection operators denoted by $\mathcal{P}\left(\mathcal{C}_{m}\right)$, i.e.,

$$
\mathcal{C}_{m}=\frac{1}{N} \sum \mathcal{P}\left(\mathcal{C}_{m}\right) \mathcal{A}_{i j} \delta_{i j},
$$


where the sum includes spin, flavors and colors of the external fermions; $N$ is the number of colors in $\mathrm{SU}(\mathrm{N})$ gauge theory. In $d$-space-time dimensions, the projectors that satisfy $\sum \mathcal{P}\left(\mathcal{C}_{m}\right) \mathcal{T}_{m}=1$ and $\sum \mathcal{P}\left(\mathcal{C}_{m}\right) \mathcal{T}_{n}=0 \forall m \neq n$, are found to be

$$
\begin{aligned}
& \mathcal{P}\left(\mathcal{C}_{1}\right)=\frac{1}{2 s} \mathcal{T}_{1}^{\dagger}, \\
& \mathcal{P}\left(\mathcal{C}_{2}\right)=\frac{1}{2\left[\left(m_{h}^{2}-t\right)\left(m_{h}^{2}-u\right)-s m_{h}^{2}\right]} \mathcal{T}_{2}^{\dagger} .
\end{aligned}
$$

Since the application of projection operators on the amplitude gives only Lorentz scalar functions, the algebraic manipulations with loop integrals become straightforward. The square of the amplitudes, that contributes to the total cross-section, can now be obtained from the coefficients $\mathcal{C}_{1}$ and $\mathcal{C}_{2}$ using

$$
\left|\mathcal{A}_{i j}\right|^{2}=N\left[\left|\mathcal{C}_{1}\right|^{2} \mathcal{T}_{1} \mathcal{T}_{1}^{\dagger}+\left|\mathcal{C}_{2}\right|^{2} \mathcal{T}_{2} \mathcal{T}_{2}^{\dagger}+\mathcal{C}_{1} \mathcal{C}_{2}^{\dagger} \mathcal{T}_{1} \mathcal{T}_{2}^{\dagger}+\mathcal{C}_{1}^{\dagger} \mathcal{C}_{2} \mathcal{T}_{2} \mathcal{T}_{1}^{\dagger}\right]
$$

Note that these coefficients are in general complex due to the Feynman loop integrals. We expand the amplitude $\mathcal{A}_{i j}$ as well as the coefficients $\mathcal{C}_{m}$ in powers of the strong coupling constant defined by $a_{s}=g_{s}^{2}\left(\mu_{R}^{2}\right) / 16 \pi^{2}$, where $g_{s}$ is the renormalized strong coupling constant and $\mu_{R}$ is the renormalization scale:

$$
\mathcal{A}_{i j}=\sum_{l=0}^{\infty} a_{s}^{l} \mathcal{A}_{i j}^{(l)}, \quad \mathcal{C}_{m}=\sum_{l=0}^{\infty} a_{s}^{l} \mathcal{C}_{m}^{(l)},
$$

and consequently

$$
\mathcal{A}_{i j}^{(l)}=\left(\mathcal{C}_{1}^{(l)} \mathcal{T}_{1}+\mathcal{C}_{2}^{(l)} \mathcal{T}_{2}\right) \delta_{i j}
$$

Our next task is to compute these coefficients $\mathcal{C}_{m}^{(l)}, m=1,2$, up to two loop level, i.e., up to $\mathcal{O}\left(a_{s}^{2}\right)$ in perturbative QCD.

\subsection{Classification of Feynman diagrams}

At LO, only three Feynman diagrams contribute, out of which one contains single Yukawa and trilinear couplings, and the remaining ones are quadratic in the Yukawa coupling. We denote the former by Class-A and the latter diagrams by Class-B. The same classes of diagrams contribute beyond LO. We elaborate on these classes of diagrams below:

- Class-A: it contains diagrams where an off-shell Higgs boson produced in the bottom quark annihilation process decays to a final state containing a pair of on-shell Higgs bosons $\left(H^{*} \rightarrow H H\right)$ and is proportional to $\lambda_{3}^{S M} \lambda_{b}$. They are shown in figure 1 . Note that the decay part of the amplitudes does not get any QCD corrections, however the initial states do get. These corrections are identical to those that contribute to the amplitudes for producing a single Higgs boson in bottom quark annihilation. The latter is known up to three-loop level in QCD [44].

- Class-B: it contains diagrams where both the Higgs bosons couple directly to the bottom quarks and hence they are proportional to $\lambda_{b}^{2}$ as shown in figure 2. At two 

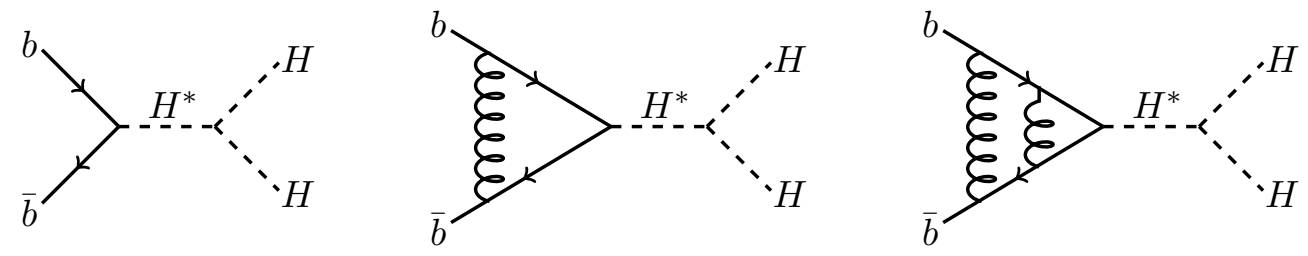

Figure 1. Illustration of Class-A diagrams; Born, one and two-loop examples.
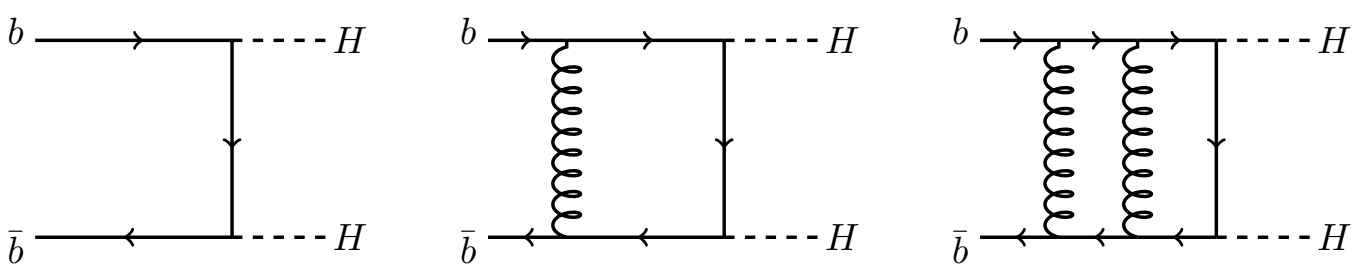

Figure 2. Illustration of Class-B diagrams; Born, one and two-loop examples.
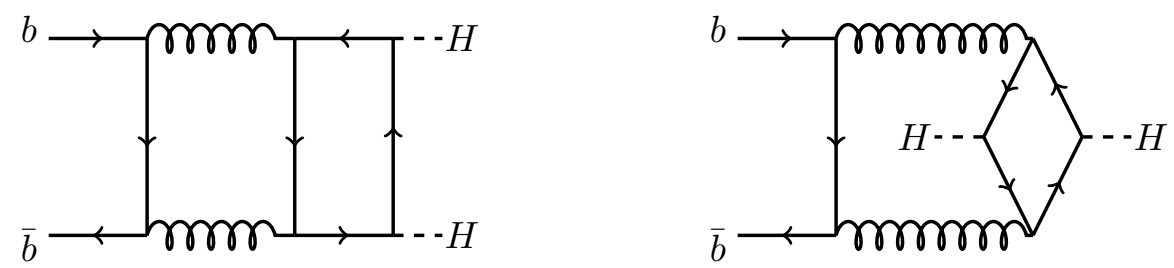

Figure 3. Illustration of special set of Class-B diagrams, the singlet contributions.

loops level, one encounters a new set of diagrams, the singlet contributions, where both the Higgs bosons are produced from a closed bottom quark loop as shown in figure 3. Here in this class of diagrams, we have dropped the dominant contributions coming from the top quark loops and computed only those from bottom quark loops as the former ones are already included in the gluon initiated subprocesses obtained in the heavy top limit in [25] for the Higgs pair production at the LHC.

\section{Methodology}

\subsection{Computational details}

It is easy to see from the form of $\mathcal{T}_{i}$ in eq. (2.8), that only the Class-A diagrams contribute to $\mathcal{C}_{1}$ and the Class-B to $\mathcal{C}_{2}$. Note that the Class-A diagrams are already computed to three loops in QCD [44]. Hence in this section, we briefly discuss how the scalar function $\mathcal{C}_{2}$ in eq. (2.9) is computed order by order in perturbation theory. As we mentioned, we use the dimensional regularization, in which the space-time dimensions are taken to be $d=4+\epsilon$ and perform traces of Dirac $\gamma$ matrices, contraction of Lorentz indices in $d$-dimensions. For convenience, we work with the bare form of the Lagrangian and evaluate the coefficient $\mathcal{C}_{2}$ in powers of bare coupling constant $\hat{a}_{s}$, where $\hat{a}_{s}=\hat{g}_{s}^{2} / 16 \pi^{2}, \hat{g}_{s}$ being the dimensionless strong coupling constant. Beyond LO, one- and two-loop amplitudes containing massless 
quarks, anti-quarks and gluons develop IR divergences in addition to UV ones. There are two types of IR divergences, viz soft and collinear divergences. The soft ones are due to soft gluons and the collinear ones arise due to massless quarks and gluons. Dimensional regularization regulates both these divergences in addition to UV divergences.

We have used QGRAF [58] to generate the Feynman diagrams at every order in the strong coupling constant. Beyond one-loop, large number of Feynman diagrams contributes to the amplitude. We find that there are 2 diagrams at the Born level, 10 diagrams at one-loop and 153 diagrams at two-loop level. We then multiply these amplitudes with the projection operator $\mathcal{P}\left(\mathcal{C}_{2}\right)$ defined in eq. (2.10) to obtain the scalar function $\mathcal{C}_{2}$. Substitution of Feynman rules and computation of various traces involving Dirac and Gell-Mann matrices, are done using in-house routines that use publicly available packages such as FORM [59] and Mathematica. At this stage we end up with a large number of one- and two-loop Feynman integrals. The projection operators guarantee that all the tensor integrals are converted to scalar integrals. We rearrange all the Feynman integrals into a few chosen integral families through shifting of loop momentum. To achieve this, we use the package Reduze2 [60]. At one-loop, the following three integral families can accommodate all the Feynman integrals

$$
\left\{\mathcal{P}_{1}, \mathcal{P}_{1: i}, \mathcal{P}_{1: i, i+1}, \mathcal{P}_{1: i, i+1, i+2}\right\},
$$

where, $i$ takes one of the values $\{1,2,3\}$ whose elements are arranged cyclically. A typical two-loop topology contains at most seven propagators. However, there are nine different Lorentz invariants $\left(k_{i} \cdot k_{j}, k_{i} \cdot p_{j}\right)$ which can appear in the numerator of an integral. Hence, we introduce two auxiliary propagators in each of the two-loop integral families. The following two sets describe the six integral families that we use at two-loops,

$$
\begin{aligned}
& \left\{\mathcal{P}_{0}, \mathcal{P}_{1}, \mathcal{P}_{2}, \mathcal{P}_{1: i}, \mathcal{P}_{2: i}, \mathcal{P}_{1: i, i+1}, \mathcal{P}_{2: i, i+1} \mathcal{P}_{1: i, i+1, i+2}, \mathcal{P}_{2: i, i+1, i+2}\right\} \\
& \left\{\mathcal{P}_{0}, \mathcal{P}_{1}, \mathcal{P}_{2}, \mathcal{P}_{1: i}, \mathcal{P}_{2: i}, \mathcal{P}_{1: i, i+1}, \mathcal{P}_{2: i, i+1}, \mathcal{P}_{0: i+2}, \mathcal{P}_{1: i, i+1, i+2}\right\}
\end{aligned}
$$

Here,

$$
\begin{aligned}
\mathcal{P}_{\alpha} & =k_{\alpha}^{2}, & \mathcal{P}_{\alpha: i} & =\left(k_{\alpha}-p_{i}\right)^{2}, \\
\mathcal{P}_{\alpha: i j} & =\left(k_{\alpha}-p_{i}-p_{j}\right)^{2}, & \mathcal{P}_{\alpha: i j k} & =\left(k_{\alpha}-p_{i}-p_{j}-p_{k}\right)^{2}, \\
\mathcal{P}_{0} & =\left(k_{1}-k_{2}\right)^{2}, & \mathcal{P}_{0: i} & =\left(k_{1}-k_{2}-p_{i}\right)^{2} .
\end{aligned}
$$

This large number of Feynman integrals belonging to different integral families can be written in terms of a smaller set of integrals, so-called master integrals (MIs). This can be achieved by using the integration-by-parts (IBP) [61, 62] and the Lorentz Invariance (LI) [63] identities, which are implemented in the Mathematica based package LiteRed [64]. Finally, we obtain 10 and 149 MIs at one- and two-loops, respectively. The resulting set of MIs is systematically mapped on to those evaluated in $[57,65]$ as Laurent series in $\epsilon$ up to the required order. Finally, substituting the results of MIs from [57, 65], we obtain the two-loop result for the coefficient $\mathcal{C}_{2}$. Both UV and IR divergences appear as poles in $\epsilon$ at every order in $\hat{a}_{s}$. In the next section, we demonstrate how the renormalization of the strong and the Yukawa couplings render these coefficients UV finite leaving only IR divergences. 


\section{$3.2 \quad$ Ultraviolet renormalization}

The scalar function $\mathcal{C}_{2}$ computed in powers of the bare coupling constant $\hat{a}_{s}$ contains both UV and IR divergences. Note that the entire amplitude is proportional to the square of $\hat{\lambda}_{b}$, the bare Yukawa coupling. We use the modified minimal subtraction $(\overline{M S})$ scheme to perform the UV renormalization of the amplitudes. In this scheme, the renormalized strong coupling constant $a_{s}$ is related to the bare strong coupling constant, $\hat{a}_{s}$, through the renormalization constant $Z\left(a_{s}\left(\mu_{R}^{2}\right), \epsilon\right)$ at the renormalization scale $\mu_{R}$ as

$$
\frac{\hat{a}_{s}}{\mu_{0}^{\epsilon}} S_{\epsilon}=\frac{a_{s}}{\mu_{R}^{\epsilon}} Z\left(a_{s}\left(\mu_{R}^{2}\right), \epsilon\right),
$$

where $Z\left(a_{s}\left(\mu_{R}^{2}\right), \epsilon\right)$ up to two-loops is given by

$$
Z\left(a_{s}\left(\mu_{R}^{2}\right), \epsilon\right)=1+a_{s}\left(\frac{2 \beta_{0}}{\epsilon}\right)+a_{s}^{2}\left(\frac{4 \beta_{0}^{2}}{\epsilon^{2}}+\frac{\beta_{1}}{\epsilon}\right)+\mathcal{O}\left(a_{s}^{3}\right) .
$$

Here, $S_{\epsilon} \equiv \exp \left[\left(\gamma_{E}-\ln 4 \pi\right) \frac{\epsilon}{2}\right]$ is the phase-space factor in $d$-dimensions, $\gamma_{E}=0.5772 \ldots$ is the Euler-Mascheroni constant and $\mu_{0}$ is an arbitrary mass scale introduced to make $\hat{g}_{s}$ dimensionless in $d$-dimensions. The constants $\beta_{0}$ and $\beta_{1}$ are the coefficients of $\beta$ function which, for $n_{f}$ light quark flavors, are found [66-70] to be

$$
\beta_{0}=\frac{11}{3} C_{A}-\frac{4}{3} n_{f} T_{F}, \quad \beta_{1}=\frac{34}{3} C_{A}^{2}-\frac{20}{3} C_{A} n_{f} T_{F}-4 C_{F} n_{f} T_{F},
$$

where $C_{A}=N, C_{F}=\left(N^{2}-1\right) / 2 N$ are the Casimirs of SU(N) group and $T_{F}=1 / 2$. Similar to $\hat{a}_{s}$, the renormalization of the Yukawa coupling constant $\hat{\lambda}_{b}$ leads to renormalized $\lambda_{b}\left(\mu_{R}^{2}\right)$ at the renormalization scale $\mu_{R}$ through

$$
\begin{aligned}
\frac{\hat{\lambda}_{b}}{\mu_{0}^{\epsilon / 2}} S_{\epsilon} & =\frac{\lambda_{b}}{\mu_{R}^{\epsilon / 2}} Z_{\lambda}\left(a_{s}\left(\mu_{R}^{2}\right), \epsilon\right) \\
& =\frac{\lambda_{b}}{\mu_{R}^{\epsilon / 2}}\left[1+a_{s}\left(\frac{1}{\epsilon} Z_{\lambda, 1}^{(1)}\right)+a_{s}^{2}\left(\frac{1}{\epsilon^{2}} Z_{\lambda, 2}^{(2)}+\frac{1}{\epsilon} Z_{\lambda, 1}^{(2)}\right)+\mathcal{O}\left(a_{s}^{3}\right)\right]
\end{aligned}
$$

where the coefficients $Z_{\lambda, j}^{(i)}$ are given by

$$
Z_{\lambda, 1}^{(1)}=6 C_{F}, Z_{\lambda, 2}^{(2)}=18 C_{F}^{2}+6 \beta_{0} C_{F}, Z_{\lambda, 1}^{(2)}=\frac{3}{2} C_{F}^{2}+\frac{97}{6} C_{F} C_{A}-\frac{10}{3} C_{F} n_{f} T_{F} .
$$

The perturbative expansion of the amplitude for the aforementioned process in terms of the bare strong and Yukawa couplings is given by

$$
\mathcal{A}_{i j}=\left(\frac{\hat{\lambda}_{b}}{\mu_{0}^{\epsilon / 2}} S_{\epsilon}\right)^{2}\left[\hat{\mathcal{A}}_{i j}^{(0)}+\left(\frac{\hat{a}_{s}}{\mu_{0}^{\epsilon}} S_{\epsilon}\right) \hat{\mathcal{A}}_{i j}^{(1)}+\left(\frac{\hat{a}_{s}}{\mu_{0}^{\epsilon}} S_{\epsilon}\right)^{2} \hat{\mathcal{A}}_{i j}^{(2)}+\mathcal{O}\left(\hat{a}_{s}^{3}\right)\right],
$$

where $\hat{\mathcal{A}}_{i j}^{(l)}$ is the $l^{\text {th }}$ loop unrenormalized amplitude. Similarly, the coefficient $\mathcal{C}_{2}$ replicates similar perturbative expansion of the following form,

$$
\mathcal{C}_{2}=\left(\frac{\hat{\lambda}_{b}}{\mu_{0}^{\epsilon / 2}} S_{\epsilon}\right)^{2}\left[\hat{\mathcal{C}}_{2}^{(0)}+\left(\frac{\hat{a}_{s}}{\mu_{0}^{\epsilon}} S_{\epsilon}\right) \hat{\mathcal{C}}_{2}^{(1)}+\left(\frac{\hat{a}_{s}}{\mu_{0}^{\epsilon}} S_{\epsilon}\right)^{2} \hat{\mathcal{C}}_{2}^{(2)}+\mathcal{O}\left(\hat{a}_{s}^{3}\right)\right]
$$


In terms of the renormalized couplings, the coefficient $\mathcal{C}_{2}$ takes the form

$$
\mathcal{C}_{2}=\left(\frac{\lambda_{b}}{\mu_{R}^{\epsilon / 2}}\right)^{2}\left[\mathcal{C}_{2}^{(0)}+a_{s} \mathcal{C}_{2}^{(1)}+a_{s}^{2} \mathcal{C}_{2}^{(2)}+\mathcal{O}\left(a_{s}^{3}\right)\right]
$$

We obtain the coefficients $\mathcal{C}_{2}^{(i)}$ using eq. (3.3) and (3.6) in eq. (3.9) and comparing with eq. (3.10):

$$
\begin{aligned}
\mathcal{C}_{2}^{(0)}= & \hat{\mathcal{C}}_{2}^{(0)} \\
\mathcal{C}_{2}^{(1)}= & \frac{12}{\epsilon} C_{F} \hat{\mathcal{C}}_{2}^{(0)}+\frac{1}{\mu_{R}^{\epsilon}} \hat{\mathcal{C}}_{2}^{(1)}, \\
\mathcal{C}_{2}^{(2)}= & {\left[\frac{12}{\epsilon^{2}}\left(6 C_{F}^{2}+\beta_{0} C_{F}\right)+\frac{1}{\epsilon}\left(3 C_{F}^{2}+\frac{97}{3} C_{F} C_{A}-\frac{20}{3} C_{F} n_{f} T_{F}\right)\right] \hat{\mathcal{C}}_{2}^{(0)} } \\
& +\frac{2}{\mu_{R}^{\epsilon}}\left[\frac{\beta_{0}}{\epsilon}+\frac{6 C_{F}}{\epsilon}\right] \hat{\mathcal{C}}_{2}^{(1)}+\frac{1}{\mu_{R}^{2 \epsilon}} \hat{\mathcal{C}}_{2}^{(2)} .
\end{aligned}
$$

These constants $\mathcal{C}_{2}^{(l)}, l=0,1,2$, that result after performing the renormalization of the strong and the Yukawa couplings, are UV finite. However they are sensitive to both soft and collinear divergences which will be the topic of our next section. These soft and collinear divergences show up in terms of poles in $\epsilon$.

\subsection{Infrared divergences and their factorization}

The UV finite amplitudes still contain divergences resulting from soft and collinear regions of the loop integrals. They result from soft gluons and massless collinear quarks and gluons in the loops. In the physical observables, the soft and the collinear divergences coming from the final states of the virtual diagrams cancel against those resulting from the phase space integrals of the real emission processes. Due to the Kinoshita-Lee-Nauenberg (KLN) theorem $[71,72]$, the cancellation takes place order by order in perturbation theory. While the soft divergences cancel fully, the collinear divergences resulting from initial massless states, do not cancel at the sub-process level. Thanks to the collinear factorization theorem [73] these initial state collinear divergences can be factored out in a process independent way and absorbed into the bare parton distribution functions. This procedure is called mass factorization which is also a consequence of KLN theorem applied at the hadronic level. While all these IR divergences that appear in the amplitudes do not pose any problem for the physical observables, they provide valuable information about the universal structure of the infrared divergences in the QCD amplitudes. In fact, it can be shown that these divergences systematically factor out from the amplitudes to all orders in perturbation theory $[74,75]$. These factored IR divergences demonstrate the universal structure in terms of certain soft and collinear anomalous dimensions. An elegant proposal was put forth by Catani who predicted IR pole structure of the amplitudes up to two-loop level in non-abelian gauge theory [55]. He demonstrated that the $n$-particle QCD amplitudes factorize in terms of the universal IR subtraction operator denoted by $\mathcal{I}$. This $\mathcal{I}$-operator has a dipole structure [55] containing process independent universal cusp and collinear 
anomalous dimensions. Thanks to the wealth of results from two-loop calculations of the three-parton $q \bar{q} g$ amplitudes [76] and $2 \rightarrow 2$ scattering amplitudes [77-79], that involve nontrivial color structures $[79,80]$, the $\mathcal{I}$-operator is completely known up to two-loop level. In [81], the authors provide further insight on the factorization and resummation properties of QCD amplitudes in the light of Catani's proposal and demonstrate a connection between divergences governed by soft and collinear anomalous dimensions, see also [82, 83]. There have been several efforts $[84,85]$ to determine the structure of $\mathcal{I}$-operator beyond two-loop level. Following [55] we express one and two-loop UV renormalized amplitudes in terms of the $\mathcal{I}$-operator as

$$
\begin{aligned}
& \mathcal{C}_{2}^{(0)}(\epsilon)=\mathcal{C}_{2}^{(0), \text { fin }}(\epsilon) \\
& \mathcal{C}_{2}^{(1)}(\epsilon)=2 \mathcal{I}_{b}^{(1)}(\epsilon) \mathcal{C}_{2}^{(0)}(\epsilon)+\mathcal{C}_{2}^{(1), \text { fin }}(\epsilon) \\
& \mathcal{C}_{2}^{(2)}(\epsilon)=4 \mathcal{I}_{b}^{(2)}(\epsilon) \mathcal{C}_{2}^{(0)}(\epsilon)+2 \mathcal{I}_{b}^{(1)}(\epsilon) \mathcal{C}_{2}^{(1)}(\epsilon)+\mathcal{C}_{2}^{(2), \text { fin }}(\epsilon)
\end{aligned}
$$

The matrix elements of the subtraction operator for the bottom quark, $\mathcal{I}_{b}$ are given by

$$
\begin{aligned}
& \mathcal{I}_{b}^{(1)}(\epsilon)=\frac{e^{-\frac{\epsilon}{2} \gamma_{E}}}{\Gamma(1+\epsilon / 2)}\left(-\frac{4 C_{F}}{\epsilon^{2}}+\frac{3 C_{F}}{\epsilon}\right)\left(-\frac{s}{\mu_{R}^{2}}\right)^{\frac{\epsilon}{2}}, \\
& \mathcal{I}_{b}^{(2)}(\epsilon)=-\frac{1}{2} \mathcal{I}_{b}^{(1)}(\epsilon)\left(\mathcal{I}_{b}^{(1)}(\epsilon)-\frac{2 \beta_{0}}{\epsilon}\right)+\frac{e^{\frac{\epsilon}{2} \gamma_{E}} \Gamma(1+\epsilon)}{\Gamma(1+\epsilon / 2)}\left(-\frac{\beta_{0}}{\epsilon}+K\right) \mathcal{I}_{b}^{(1)}(2 \epsilon)+2 H_{b}^{(2)}(\epsilon),
\end{aligned}
$$

with $K[55]$ and $H_{b}^{(2)}[81]$ given by as follows

$$
\begin{aligned}
& K=\left(\frac{67}{18}-\frac{\pi^{2}}{6}\right) C_{A}-\frac{10}{9} n_{f} T_{F}, \\
& H_{b}^{(2)}=\left(-\frac{s}{\mu_{R}^{2}}\right)^{\epsilon} \frac{e^{-\frac{\epsilon}{2} \gamma_{E}}}{\Gamma(1+\epsilon / 2)} \frac{1}{\epsilon}\left[C_{A} C_{F}\left(-\frac{245}{432}+\frac{23}{16} \zeta_{2}-\frac{13}{4} \zeta_{3}\right)\right. \\
& \left.+C_{F}^{2}\left(\frac{3}{16}-\frac{3}{2} \zeta_{2}+3 \zeta_{3}\right)+C_{F} n_{f}\left(\frac{25}{216}-\frac{1}{8} \zeta_{2}\right)\right] .
\end{aligned}
$$

According to the proposal by Catani, the coefficients $\mathcal{C}_{2}^{(i), \text { fin }}(\epsilon)$ should be free of IR divergences and hence are finite as $\epsilon \rightarrow 0$. Since the resulting expression at two-loops level, $\mathcal{C}_{2}^{(2), \text { fin }}(\epsilon)$ is quiet lengthy, we had to simplify the expression first at the color factor level and then for each color factor, terms of uniform transcendentality were further simplified. We find that our final result is in accordance with Catani's predictions for the IR poles, which serves as an important check on the correctness of our computation.

It is interesting to observe that the singlet contributions which are proportional to the color factor $C_{F} n_{b} T_{F}$, for $n_{b}=1$, develops IR divergences at the intermediate stages of the computation. However at the end, all the IR singularities cancel among themselves contributing only to the IR finite part. This is consistent with the IR pole structure predicted by Catani. The resulting finite constant $\mathcal{C}_{2}^{(2) \text {,fin }}$ that results after subtracting the IR divergences using Catani's $\mathcal{I}$-operators is too lengthy to be presented here and hence attached as supplementary material in Mathematica format. 


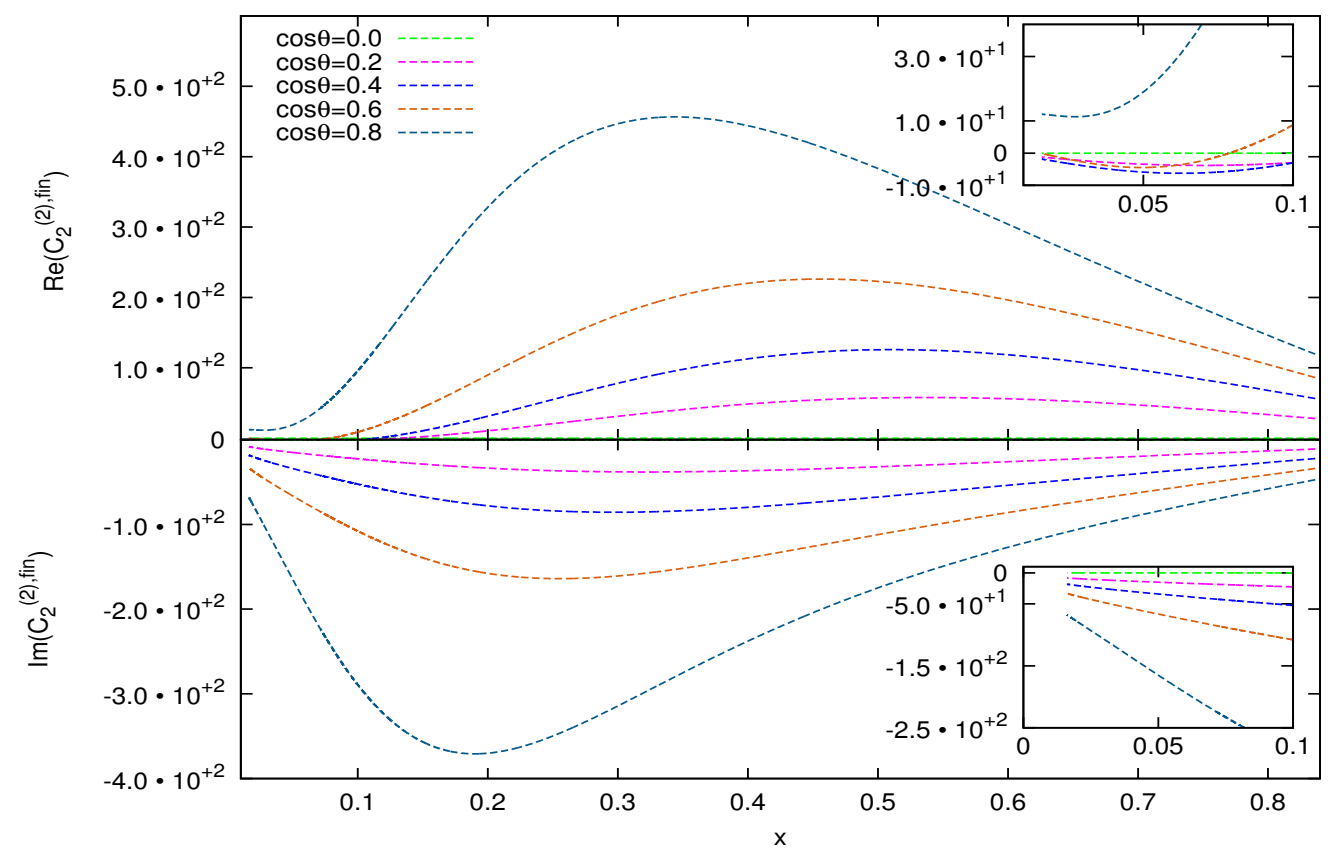

Figure 4. Behaviour of the real and the imaginary part of $\mathcal{C}_{2}^{(2), f i n}$ as a function of the scaling variable $x$ for different values of $\cos (\theta)$. The insets show the region close to $x=0$.

\section{Numerical evaluation of the amplitude}

The finite coefficients, $\mathcal{C}_{2}^{(i), \text { fin }}, i=1,2$, obtained in eq. (3.12) contain multiple classical polylogarithms, which are functions of the scaling variables $x$ and $y$. These polylogarithms can be attributed to different transcendental weights, the property that we use to simplify the two loop coefficient, $\mathcal{C}_{2}^{(2), \text { fin }}$. Considering the complexity of our final result $\mathcal{C}_{2}^{(2) \text {,fin }}$, we perform a numerical evaluation using Mathematica for a wide range of scaling variables. More precisely, in figure 4 we plot the real and the imaginary parts of the coefficient $\mathcal{C}_{2}^{(2) \text {,fin }}$ as functions of the scaling variable $x$ for different values of $\cos (\theta)$, where $\theta$ denotes the angle between one of the initial state fermions and the Higgs boson in the center of mass frame of incoming states. We consider $m_{h}=125 \mathrm{GeV}$ and the renormalization scale as $\mu_{R}^{2}=m_{h}^{2} / 2$. In addition, we normalize the coefficient with the factor $m_{h}^{2}$. The amplitude is anti-symmetric under $\cos (\theta) \rightarrow-\cos (\theta)$, as expected for a purely fermionic amplitude. Since this symmetry has not been used in the setup of the calculation, it serves as a strong check on our results. Our expression contains polylogarithms that are multiplied by large rational coefficients, hence we encounter numerical instabilities during the evaluation. To avoid this, we evaluate the polylogarithms at double precision while setting the rational coefficients at higher precision. From the figure 4, we observe a stable behaviour for the real and imaginary parts for the range of parameters considered. In addition, the dependence of the coefficient near the phase space boundary $x=0$ is displayed in the insets. The simplified analytical results and their numerical implementation are provided as supplementary material. 


\section{Inclusive cross section up to NNLO}

In this section, we describe in detail the computation of inclusive cross section up to NNLO level for producing a pair of Higgs bosons resulting class-A and class-B diagrams. The hadronic cross section can be expressed in terms of partonic cross sections appropriately convoluted with the corresponding bare parton distribution functions $\hat{f}_{a_{i}}\left(x_{i}\right), i=1,2$ as

$$
\sigma^{H H}=\sum_{a_{1}, a_{2}} \int d x_{1} \hat{f}_{a_{1}}\left(x_{1}\right) \int d x_{2} \hat{f}_{a_{2}}\left(x_{2}\right) \hat{\sigma}_{a_{1} a_{2}}^{H H}\left(x_{1}, x_{2}, m_{h}^{2}\right),
$$

where $x_{i}$ are the momentum fractions of initial state partons and $a_{1,2}=q, \bar{q}, g . \hat{\sigma}_{a_{1} a_{2}}^{H H}$ is the UV finite partonic cross section for producing a pair of Higgs bosons along with $n_{X}$ number of colored particles (partons) through the reactions $a_{1}\left(p_{1}\right)+a_{2}\left(p_{2}\right) \rightarrow H\left(q_{1}\right)+H\left(q_{2}\right)+X\left(k_{c}\right)$ and is obtained using

$$
\hat{\sigma}_{a_{1} a_{2}}^{H H}=\frac{1}{2 s} \prod_{n=1}^{2} \int d \phi\left(q_{n}\right) \prod_{c=1}^{n_{X}} \int d \phi\left(k_{c}\right) \bar{\sum}\left|\mathcal{M}_{a_{1} a_{2}}\right|^{2}(2 \pi)^{d} \delta^{d}\left(p_{1}+p_{2}-\sum_{n=1}^{2} q_{n}-\sum_{c=1}^{n_{X}} k_{c}\right)
$$

where $p_{i}, q_{i}$ and $k_{c}$ are the momenta of incoming partons, final state Higgs bosons and partons respectively. In $d$-dimensions, the phase space measure $d \phi(p)$ of a final state particle with momentum $p$ and mass $m$ is given by

$$
d \phi(p)=\frac{d^{d-1} \vec{p}}{(2 \pi)^{d-1} 2 p^{0}}
$$

where $p^{0}=\sqrt{m^{2}+|\vec{p}|^{2}} \cdot \mathcal{M}_{a_{1} a_{2}}$ is the amplitude for the process $a_{1}\left(p_{1}\right)+a_{2}\left(p_{2}\right) \rightarrow H\left(q_{1}\right)+$ $H\left(q_{2}\right)+X\left(k_{c}\right)$ and is calculable order by order in perturbative QCD. The symbol $\bar{\sum}$ indicates that we have to sum over all the quantum numbers of final states, average over initial states and finally include the symmetry factor for final state identical particles. For convenience, we classify the partonic channels that contribute to $\mathcal{M}_{a_{1} a_{2}}$ into class-A and class-B. We find that these channels do not interfere for the inclusive cross section as well as for the invariant mass distribution of Higgs boson pairs. Hence, in the following we treat them separately.

For the class-A diagrams, the amplitude $\mathcal{M}_{a_{1} a_{2}}$ factorises into a product of two sub amplitudes, where one of them describes the production of a single Higgs boson with virtuality $Q^{2}$ and the other encapsulates its decay to a pair of on-shell Higgs bosons. By suitably factorising the phase space we can describe the entire reaction as a continuous process of producing a single off-shell boson with different virtualities, subsequently decaying to a pair of on-shell Higgs bosons. In other words, we can write $\hat{\sigma}_{a_{1} a_{2}}^{H H}$ for class-A diagrams as

$$
\hat{\sigma}_{A, a_{1} a_{2}}^{H H}=\int \frac{d Q^{2}}{2 \pi} \hat{\sigma}_{A, a_{1} a_{2}}^{H^{*}}\left(x_{1}, x_{2}, Q^{2}\right)\left|\mathcal{P}_{H}\left(Q^{2}\right)\right|^{2} 2 Q \Gamma_{A}^{H^{*} \rightarrow H H}\left(Q^{2}\right)
$$

where the $\mathcal{P}_{H}\left(Q^{2}\right)$ is the Higgs boson propagator, given by

$$
\mathcal{P}_{H}\left(Q^{2}\right)=\frac{i}{Q^{2}-m_{h}^{2}+i \Gamma_{h} m_{h}}
$$


with $\Gamma_{h}$, the decay width of the Higgs boson. The cross section that describes the production of a Higgs boson with virtuality $Q^{2}$ is given by

$$
\begin{aligned}
\hat{\sigma}_{A, a_{1} a_{2}}^{H^{*}}\left(x_{1}, x_{2}, Q^{2}\right)= & \frac{1}{2 s} \prod_{c=1}^{n_{X}} \int d \phi\left(k_{c}\right) \\
& \times \int d \phi(Q) \bar{\sum}\left|\mathcal{M}_{A, a_{1} a_{2}}^{H^{*}}\right|^{2}(2 \pi)^{d} \delta^{d}\left(p_{1}+p_{2}-Q-\sum_{c=1}^{n_{X}} k_{c}\right) .
\end{aligned}
$$

Here $\mathcal{M}_{A, a_{1} a_{2}}^{H^{*}}$ is the amplitude for the production of an off-shell Higgs boson with the virtuality $Q^{2}$ and $n_{X}$ number of colored particles. The decay rate $\Gamma_{A}^{H^{*} \rightarrow H H}$ is given by

$$
\Gamma_{A}^{H^{*} \rightarrow H H}\left(Q^{2}\right)=\frac{1}{2 Q} \prod_{n=1}^{2} \int d \phi\left(q_{n}\right) \bar{\sum}\left|\mathcal{M}_{A}^{H^{*} \rightarrow H H}\right|^{2}(2 \pi)^{d} \delta^{d}\left(Q-\sum_{n=1}^{2} q_{n}\right),
$$

with $\mathcal{M}_{A}^{H^{*} \rightarrow H H}$ describing its decay into a pair of on-shell Higgs bosons. The decay rate $\Gamma_{A}^{H^{*} \rightarrow H H}$ is straightforward to compute and in 4-dimensions it is found to be

$$
\Gamma_{A}^{H^{*} \rightarrow H H}\left(Q^{2}\right)=\frac{9 \beta\left(Q^{2}\right) m_{h}^{4}}{32 \pi v^{2} Q}, \quad \beta\left(Q^{2}\right)=\sqrt{1-\frac{4 m_{h}^{2}}{Q^{2}}} .
$$

Substituting eq. (5.4) in eq. (5.1) and using eqs. (5.6), (5.7), we obtain $\sigma_{A}^{H H}$, the contribution of class-A diagrams to $\sigma^{H H}$ in eq. (5.2):

$$
\sigma_{A}^{H H}=\int \frac{d Q^{2}}{2 \pi} D_{H}\left(Q^{2}\right) \sigma_{A}^{H^{*}}\left(Q^{2}\right)
$$

with

$$
\sigma_{A}^{H^{*}}\left(Q^{2}\right)=\sum_{a_{1}, a_{2}} \int d x_{1} \hat{f}_{a_{1}}\left(x_{1}\right) \int d x_{2} \hat{f}_{a_{2}}\left(x_{2}\right) \hat{\sigma}_{A, a_{1} a_{2}}^{H^{*}}\left(z, Q^{2}\right)
$$

where the partonic scaling variable $z=Q^{2} / s$ and $D_{H}\left(Q^{2}\right)=2 Q \Gamma_{A}^{H^{*} \rightarrow H H}\left(Q^{2}\right)\left|\mathcal{P}_{H}\left(Q^{2}\right)\right|^{2}$. Note that $\sigma_{A}^{H^{*}}$ is known exactly up to NNLO level [42] and $\mathrm{N}^{3} \mathrm{LO}$ level $[45,56]$ in the soft plus virtual approximation for on-shell production of single Higgs boson. Hence, following [42], we can express $\sigma_{A}^{H^{*}}\left(Q^{2}\right)$ in terms of IR finite coefficients convoluted with renormalized parton distribution functions $f_{c}\left(x, \mu_{F}\right)$ as

$$
\sigma_{A}^{H^{*}}\left(Q^{2}\right)=\sigma_{0}^{H^{*}}\left(Q^{2}, \mu_{R}\right) \sum_{a_{1}, a_{2}} \int d x_{1} f_{a_{1}}\left(x_{1}, \mu_{F}\right) \int d x_{2} f_{a_{2}}\left(x_{2}, \mu_{F}\right) z \Delta_{A, a_{1} a_{2}}\left(z, Q^{2}, \mu_{F}, \mu_{R}\right)
$$

where $\sigma_{0}^{H^{*}}\left(Q^{2}, \mu_{R}\right)=\pi m_{b}^{2}\left(\mu_{R}^{2}\right) /\left(6 Q^{2} v^{2}\right) . \quad \Delta_{A, a_{1} a_{2}}$ can be expanded in powers of strong coupling constant as

$$
\Delta_{A, a_{1} a_{2}}\left(z, Q^{2}, \mu_{F}, \mu_{R}\right)=\sum_{i=0}^{\infty} a_{s}^{i}\left(\mu_{R}^{2}\right) \Delta_{A, a_{1} a_{2}}^{(i)}\left(z, Q^{2}, \mu_{F}, \mu_{R}\right) .
$$


Substituting eq. (5.11) in eq. (5.9) and making suitable change of variables, we obtain

$$
\sigma_{A}^{H H}=\sum_{a_{1} a_{2}} \int_{\tau}^{1} \frac{d x}{2 \pi} \Phi_{a_{1} a_{2}}\left(x, \mu_{F}\right) \int_{\frac{\tau}{x}}^{1} d z\left[\sigma_{0}^{H^{*}}\left(Q^{2}, \mu_{R}\right) D_{H}\left(Q^{2}\right) \Delta_{A, a_{1} a_{2}}\left(z, Q^{2}, \mu_{F}, \mu_{R}\right)\right]_{Q^{2}=x z S}
$$

where $\tau=4 m_{h}^{2} / S, S=s / x_{1} x_{2}$, the hadronic center of mass energy of incoming hadrons and the partonic flux $\Phi_{a_{1} a_{2}}\left(x, \mu_{F}\right)$ is given by

$$
\Phi_{a_{1} a_{2}}\left(x, \mu_{F}\right)=\int_{x}^{1} \frac{d y}{y} f_{a_{1}}\left(y, \mu_{F}\right) f_{a_{2}}\left(\frac{x}{y}, \mu_{F}\right) .
$$

In the next section, we use eq. (5.13) to obtain the numerical impact of class-A diagrams to the inclusive production cross section.

We now describe how the contributions to eq. (5.1) from class-B diagrams can be obtained. Since class-B diagrams contain, in addition, t and $\mathrm{u}$ channels, the corresponding amplitudes do not factorise like class-A diagrams, making the computation technically more challenging beyond NLO level. However, one can obtain certain dominant contributions of class-B processes resulting from soft gluon emission as they are process independent. Using the contributions from soft gluons, obtained in [56], and those from the two-loop virtual processes computed in the previous sections, we can readily calculate the soft plus virtual contribution up to NNLO level, a first step towards obtaining the full NNLO contribution from class-B.

For the class-B, the leading order contribution results from the Born process $b+\bar{b} \rightarrow$ $H+H$ contain $t$ and $u$ channels. At NLO, one loop virtual corrections to Born and real emission processes $b+\bar{b} \rightarrow H+H+g$ and $b(\bar{b})+g \rightarrow H+H+b(\bar{b})$ contribute. The UV divergences that are present in the virtual processes to Born processes are removed using $\overline{M S}$ renormalisation scheme. The soft and final state collinear divergences in both virtual as well as real emission processes cancel among each other while the initial state collinear divergences are factored out and absorbed into bare bottom quark densities in the $\overline{M S}$ scheme through the mass factorization. For the sub-process $b(\bar{b})+g \rightarrow H+H+b(\bar{b})$, we encounter only collinear divergences and they are removed by mass factorization. We achieve this by using the semi analytical method, namely the two cut off phase space slicing [86]. The first computation of NLO correction to production of a pair of Higgs bosons in bottom quark annihilation process was achieved in [51] by using the same approach. In the present article, we use this approach only for the class-B diagrams to compute NLO contributions. In this method, for $b+\bar{b} \rightarrow H+H+g$, two slicing parameters $\delta_{s}$ and $\delta_{c}$ are introduced to separate three body phase space into soft, hard collinear and hard non-collinear regions, while for $g+b(\bar{b}) \rightarrow H+H+b(\bar{b})$, we need to introduce only $\delta_{c}$ as these are free from soft divergences. The slicing parameter $\delta_{s}$ divides the real emission phase space into soft and hard regions. The soft region is the part of the phase space where the energy of the gluon in the center-of-mass frame of incoming partons is required to be less than $\delta_{s} \sqrt{s} / 2$ and the rest is called hard region. The later contains collinear configurations where the two massless partons become collinear to each other leading to collinear singularities. We use $\delta_{c}$ to divide the hard region into hard-collinear and hard noncollinear regions, denoted respectively by $\mathcal{H C}$ and $\overline{\mathcal{H C}}$. Keeping these slicing parameters $\delta_{s}$ 
and $\delta_{c}$ infinitesimally small, the virtual loop integrals and the soft and collinear sensitive phase space integrals are computed within the method of dimensional regularization. The corresponding singularities show up as poles in dimensional regularization parameter $\epsilon$.

We describe below the essential steps that are followed in dealing with IR singularities in phase space slicing method. We start with UV finite hadronic cross section at NLO level, denoted by $d \sigma^{H+1}$. It gets contribution from real emission partonic sub-process $a_{1}+a_{2} \rightarrow H H+a_{3}$ where the final state consists of a pair of Higgs bosons $H H$ and $a_{3}$, a single partonic state. We divide the phase space of $a_{3}$ into three regions using two slicing parameters as

$$
d \sigma^{H H+1}\left(\delta_{s}, \delta_{c}, \epsilon\right)=d \sigma^{H H, \mathcal{S}}\left(\delta_{s}, \epsilon\right)+d \sigma^{H H, \mathcal{H C}}\left(\delta_{s}, \delta_{c}, \epsilon\right)+d \sigma^{H H, \overline{\mathcal{H C}}}\left(\delta_{s}, \delta_{c}\right) .
$$

The soft $\left(d \sigma^{H H, \mathcal{S}}\left(\delta_{s}, \epsilon\right)\right)$ and hard-collinear $\left(d \sigma^{H H, \mathcal{H C}}\left(\delta_{s}, \delta_{c}, \epsilon\right)\right)$ contributions can be computed analytically when the slicing parameters are infinitesimally small within the dimensional regularization. Soft and collinear singularities appear as poles in $\epsilon$ and are cancelled against those resulting from the virtual diagrams as well as from the counter terms that are used to perform mass factorization. In other words, the following sum, denoted by $d \sigma_{\mathrm{NLO}}^{H H}$ is finite as $\epsilon \rightarrow 0$ :

$$
d \sigma_{\mathrm{NLO}}^{H H}\left(\mu_{F}\right)=d \sigma^{H H, \mathcal{V}}(\epsilon)+d \sigma^{H H+1}\left(\delta_{s}, \delta_{c}, \epsilon\right)+d \sigma^{H H, \mathrm{CT}}\left(\delta_{s}, \delta_{c}, \epsilon, \mu_{F}\right)
$$

where $d \sigma^{H H, \mathcal{V}}(\epsilon)$ is the contribution from virtual corrections to Born level processes. The counter term $d \sigma^{H H, \mathrm{CT}}\left(\delta_{s}, \delta_{c}, \epsilon, \mu_{F}\right)$ that removes the initial state collinear singularities is defined at the factorization scale $\mu_{F}$. While the sum given by

$$
\begin{aligned}
d \sigma^{\mathcal{S}+\mathcal{V}+\mathcal{H C}+\mathrm{CT}}\left(\delta_{s}, \delta_{c}, \mu_{F}\right)= & d \sigma^{H H, \mathcal{S}}\left(\delta_{s}, \epsilon\right)+d \sigma^{H H, \mathcal{V}}(\epsilon) \\
& +d \sigma^{H H, \mathcal{H C}}\left(\delta_{s}, \delta_{c}, \epsilon\right)+d \sigma^{H H, \mathrm{CT}}\left(\delta_{s}, \delta_{c}, \epsilon, \mu_{F}\right) .
\end{aligned}
$$

is free from soft and collinear poles in $\epsilon$, it depends on the slicing parameters. However, when the above sum is added to the hard non-collinear contributions $\left(d \sigma^{H H, \overline{\mathcal{H C}}}\right)$, that is,

$$
d \sigma_{\mathrm{NLO}}^{H H}\left(\mu_{F}\right)=\lim _{\delta_{s}, \delta_{c} \rightarrow 0}\left(d \sigma^{\mathcal{S}+\mathcal{V}+\mathcal{H C}+\mathrm{CT}}\left(\delta_{s}, \delta_{c}\right)+d \sigma^{H H, \overline{\mathcal{H C}}}\left(\delta_{s}, \delta_{c}\right)\right)
$$

the resulting contribution, eq. (5.18), is guaranteed to be independent of the slicing parameters in the limit when they are taken to be infinitesimally small. For the sub-process, $g+b(\bar{b}) \rightarrow H+H+b(\bar{b})$, we encounter only collinear divergences and hence we require a single slicing parameter $\delta_{c}$ to obtain infrared safe observable.

For completeness, we present the individual contributions that are required in phase space slicing method to obtain inclusive cross section up to NLO level from class B diagrams. The virtual contribution for the sub-process initiated by $b$ and $\bar{b}$ is found to be

$$
\begin{aligned}
d \sigma^{H H, \mathcal{V}}=a_{s}\left(\mu_{F}^{2}\right)\left(\frac{s}{\mu_{F}^{2}}\right)^{\frac{\epsilon}{2}} \frac{\Gamma\left(1+\frac{\epsilon}{2}\right)}{\Gamma(1+\epsilon)} d x_{1} d x_{2}\left[C_{F}\left(-\frac{16}{\epsilon^{2}}+\frac{12}{\epsilon}\right)\right. \\
\times d \sigma_{b \bar{b}}^{H H,(0)}\left(x_{1}, x_{2}, \epsilon\right)\left(f_{b}\left(x_{1}\right) f_{\bar{b}}\left(x_{2}\right)+\left(x_{1} \leftrightarrow x_{2}\right)\right) \\
\left.+d \sigma_{b \bar{b}, \text { fin }}^{H H, \mathcal{V}}\left(x_{1}, x_{2}, \epsilon\right)\left(f_{b}\left(x_{1}\right) f_{\bar{b}}\left(x_{2}\right)+\left(x_{1} \leftrightarrow x_{2}\right)\right)\right]
\end{aligned}
$$


after setting renormalization scale $\mu_{R}=\mu_{F}$. The finite part of the virtual corrections, $d \sigma_{b \bar{b}, \mathrm{fin}}^{H H, \mathcal{V}}$ can be obtained in terms of $\mathcal{C}_{2}$ given in eq. (2.13). The soft contribution is given by

$$
\begin{aligned}
d \sigma^{H H, \mathcal{S}} \simeq & a_{s}\left(\mu_{F}^{2}\right)\left(\frac{s}{\mu_{F}^{2}}\right)^{\frac{\epsilon}{2}} \frac{\Gamma\left(1+\frac{\epsilon}{2}\right)}{\Gamma(1+\epsilon)} C_{F}\left(\frac{16}{\epsilon^{2}}+\frac{16 \ln \delta_{s}}{\epsilon}+8 \ln ^{2} \delta_{s}\right) \\
& \times\left(d \sigma_{b \bar{b}}^{H H,(0)}\left(x_{1}, x_{2}, \epsilon\right) f_{b}\left(x_{1}\right) f_{\bar{b}}\left(x_{2}\right)+\left(x_{1} \leftrightarrow x_{2}\right)\right) d x_{1} d x_{2} .
\end{aligned}
$$

The sum of hard-collinear and counter term contributions from both $b \bar{b}$ annihilation and $g b(\bar{b})$ scattering processes, is found to be:

$$
\begin{aligned}
d \sigma^{\mathcal{H C}+\mathrm{CT}}= & a_{s}\left(\mu_{F}^{2}\right)\left(\frac{s}{\mu_{F}^{2}}\right)^{\frac{\epsilon}{2}} \frac{\Gamma\left(1+\frac{\epsilon}{2}\right)}{\Gamma(1+\epsilon)} d x_{1} d x_{2} \\
& \times\left[d \sigma _ { b \overline { b } } ^ { H H , ( 0 ) } ( x _ { 1 } , x _ { 2 } , \epsilon ) \left\{\frac{1}{2} f_{\bar{b}}\left(x_{1}, \mu_{F}\right) \tilde{f}_{b}\left(x_{2}, \mu_{F}\right)+\frac{1}{2} \tilde{f}_{\bar{b}}\left(x_{1}, \mu_{F}\right) f_{b}\left(x_{2}, \mu_{F}\right)\right.\right. \\
& \left.\left.+2\left(-\frac{1}{\epsilon}+\frac{1}{2} \ln \frac{s}{\mu_{F}^{2}}\right) A_{b \rightarrow b+g} f_{\bar{b}}\left(x_{1}, \mu_{F}\right) f_{b}\left(x_{2}, \mu_{F}\right)+\left(x_{1} \leftrightarrow x_{2}\right)\right\}\right]
\end{aligned}
$$

Using the diagonal Altarelli-Parisi (AP) splitting function $P_{b b}(z)$, we find

$$
A_{b \rightarrow b+g} \equiv \int_{1-\delta_{s}}^{1} \frac{d z}{z} P_{b b}(z)=4 C_{F}\left(2 \ln \delta_{s}+\frac{3}{2}\right)
$$

and from the non-diagonal ones, we obtain

$$
\tilde{f}_{b}\left(x, \mu_{F}\right)=\int_{x}^{1-\delta_{s}} \frac{d z}{z} f_{b}\left(\frac{x}{z}, \mu_{F}\right) \tilde{P}_{b b}(z)+\int_{x}^{1} \frac{d z}{z} f_{g}\left(\frac{x}{z}, \mu_{F}\right) \tilde{P}_{b g}(z),
$$

with

$$
\tilde{P}_{i j}(z)=P_{i j}(z) \ln \left(\delta_{c} \frac{1-z}{z} \frac{s}{\mu_{F}^{2}}\right)+2 P_{i j}^{\prime}(z),
$$

where $P_{i j}^{\prime}(z)[86]$ are $\epsilon$ dependent part of AP splitting functions, that is

$$
P_{i j}(z, \epsilon)=P_{i j}(z)+\epsilon P_{i j}^{\prime}(z) .
$$

Adding all the order $a_{s}$ pieces together: the virtual cross-section $d \sigma^{H H, \mathcal{V}}$ in eq. (5.19), the soft piece $d \sigma^{H H, \mathcal{S}}$ in eq. (5.20) and the mass factorized hard-collinear contribution $d \sigma^{H H, \mathcal{H C}+\mathrm{CT}}$ as given in eq. (5.21), we find that the poles in $\epsilon$ cancel in the sum given in eq. (5.18) giving IR finite NLO contribution from class-B diagrams. In the next section, we use this semi-analytical result to study the numerical impact of class-B NLO contributions on the inclusive cross section.

Going beyond NLO for the class-B diagrams requires a dedicated computation taking into account pure virtual contributions presented in the present work, the double real and single real-virtual contributions. The inclusion of the later contributions is beyond the scope of this present work. However, we can compute the SV contribution resulting from class-B diagrams using the two-loop virtual contributions computed in the present article 
and the universal soft and collinear parts. To achieve this, we follow the general formalism presented in [56], which is applicable to both classes of diagrams.

We begin with the UV finite partonic cross section for producing a pair of Higgs bosons and $n_{X}$ partons, namely for the process $b\left(p_{1}\right)+\bar{b}\left(p_{2}\right) \rightarrow H\left(q_{1}\right)+H\left(q_{2}\right)+X\left(k_{c}\right)$,

$$
\hat{\sigma}_{b \bar{b}}=\frac{1}{2 s} \prod_{n=1}^{2} \int d \phi\left(q_{n}\right) \prod_{c=1}^{n_{X}} \int d \phi\left(k_{c}\right) \bar{\sum}\left|\mathcal{M}_{b \bar{b}}\right|^{2}(2 \pi)^{d} \delta^{d}\left(p_{1}+p_{2}-\sum_{n=1}^{2} q_{n}-\sum_{c=1}^{n_{X}} k_{c}\right)
$$

where $c$ counts the number of partons in the final state. The dominant soft gluon contributions to partonic reactions are proportional to terms such as $\delta(1-z)$ and + distributions:

$$
\mathcal{D}_{j}(z)=\left(\frac{\log ^{j}(1-z)}{1-z}\right)_{+} .
$$

Such contributions result only from bottom quark annihilation sub processes. They themselves do not constitute infrared safe observables until we include pure virtual contributions and mass factorization counter-terms. The resulting one is called SV contribution.

We briefly discuss how these contributions can be obtained. In the soft limit, it is well known that the square of the real emission partonic matrix elements factorises into hard and soft parts and similarly the phase space splits into their respective phase spaces. The soft part when combined with the pure virtual corrections and the mass factorization counter terms, will give infrared safe SV part of the cross section:

$$
\begin{aligned}
\hat{\sigma}_{b \bar{b}}^{\mathrm{SV}}= & \int \frac{d Q^{2}}{Q^{2}} \frac{1}{2 s} \prod_{n=1}^{2} \int d \phi\left(q_{n}\right) \bar{\sum}\left|\mathcal{M}_{b \bar{b}}^{(0)}\right|^{2}(2 \pi)^{d} \delta^{d}\left(p_{1}+p_{2}-\sum_{n} q_{n}\right) \\
& \times \int d \phi(Q) \prod_{c=1}^{n_{X}} \int d \phi\left(k_{c}\right) \bar{\sum}\left|\mathcal{M}^{\mathrm{SV}}\right|^{2}(2 \pi)^{d} \delta^{d}\left(p_{1}+p_{2}-Q-\sum_{c} k_{c}\right)
\end{aligned}
$$

where $\mathcal{M}_{b \bar{b}}^{(0)}$ is the Born amplitude for producing a pair of Higgs bosons in bottom quark annihilation and $\mathcal{M}^{\mathrm{SV}}$ is the SV part of amplitude $\mathcal{M}_{b \bar{b}}$. The second line of the above equation can be computed order by order in perturbation theory for any colorless state with momentum $Q$ in a process independent way as the amplitude for the production of a pair of Higgs bosons factorises out at every order. Beyond the leading order, virtual corrections to Born amplitudes and multiple soft gluon emissions both from tree level amplitudes as well as from the loop corrected amplitudes contribute to the SV. While the singularities from soft gluons cancel between real and virtual amplitudes, the initial state collinear singularities can be removed only after adding appropriate mass factorization counter terms computed in the soft limit at the factorization scale $\mu_{F}$. The resulting hadronic cross section will be free of soft and collinear singularities:

$$
\begin{aligned}
\sigma^{H H, \mathrm{SV}}= & \int \frac{d Q^{2}}{Q^{2}} \sum_{b, \bar{b}} \int d x_{1} f_{b}\left(x_{1}, \mu_{F}\right) \int d x_{2} f_{\bar{b}}\left(x_{2}, \mu_{F}\right) \frac{1}{2 s} \prod_{n=1}^{2} \int d \phi\left(q_{n}\right) \\
& \times(2 \pi)^{d} \delta^{d}\left(p_{1}+p_{2}-\sum_{n=1}^{2} q_{n}\right) \bar{\sum} \sum_{i=A, B} \Delta_{i, b \bar{b}}^{\mathrm{SV}}\left(\left\{p_{j} \cdot q_{k}\right\}, z, Q^{2}, \mu_{F}, \mu_{R}\right)
\end{aligned}
$$


where $z=Q^{2} / s, i$ runs over both the classes of diagrams. Following [56], the finite coefficients $\Delta_{i, b \bar{b}}^{\mathrm{SV}}$ can be computed order by order in perturbation theory using one and two-loop virtual amplitudes, soft distribution function and diagonal mass factorization kernels. We expand $\Delta_{i, b \bar{b}}^{\mathrm{SV}}$ in powers of strong coupling constant as,

$$
\Delta_{i, b \bar{b}}^{\mathrm{SV}}=\sum_{j=0}^{\infty} a_{s}^{j}\left(Q^{2}\right) \Delta_{i, b \bar{b}}^{\mathrm{SV},(j)}\left(Q^{2}\right)
$$

where we have set $\mu_{R}^{2}=\mu_{F}^{2}=Q^{2}$. The coefficients, $\Delta_{i, b \bar{b}}^{\mathrm{SV},(j)}$ for $j=0,1,2$ can be expressed in terms of the cusp $A_{j}^{q}$, the soft $f_{j}^{q}$ and the collinear $B_{j}^{q}$ anomalous dimensions that are present in the virtual amplitudes and in the soft distribution function [56]:

$$
\begin{aligned}
\Delta_{i, b \bar{b}}^{\mathrm{SV},(0)}= & \delta(1-z)\left|\mathcal{A}_{i, 0}^{(0)}\right|^{2} \\
\Delta_{i, b \bar{b}}^{\mathrm{SV},(1)}= & \delta(1-z)\left\{\left|\mathcal{A}_{i, 0}^{(0)}\right|^{2}\left(2 \overline{\mathcal{G}}_{1}^{q,(1)}\right)+\mathcal{A}_{i, 0}^{(1)} \mathcal{A}_{i, 0}^{\star(0)}+\mathcal{A}_{i, 0}^{(0)} \mathcal{A}_{i, 0}^{\star(1)}\right\}+\mathcal{D}_{0}\left|\mathcal{A}_{i, 0}^{(0)}\right|^{2}\left(-2 f_{1}^{q}\right) \\
& +\mathcal{D}_{1}\left|\mathcal{A}_{i, 0}^{(0)}\right|^{2}\left(4 A_{1}^{q}\right) \\
\Delta_{i, \bar{b}}^{\mathrm{SV},(2)}= & \delta(1-z)\left\{\left|\mathcal{A}_{i, 0}^{(1)}\right|^{2}+\left|\mathcal{A}_{i, 0}^{(0)}\right|^{2}\left(\overline{\mathcal{G}}_{1}^{q,(2)}+2\left(\overline{\mathcal{G}}_{1}^{q,(1)}\right)^{2}+2 \beta_{0} \overline{\mathcal{G}}_{2}^{q,(1)}-8 \zeta_{3} A_{1}^{q} f_{1}^{q}\right.\right. \\
& \left.-2 \zeta_{2}\left(f_{1}^{q}\right)^{2}-\frac{4}{5} \zeta_{2}^{2}\left(A_{1}^{q}\right)^{2}\right)+\mathcal{A}_{i, 0}^{(2)} \mathcal{A}_{i, 0}^{\star(0)}+\mathcal{A}_{i, 2}^{(1)} \mathcal{A}_{i,-2}^{\star(1)} \\
& +\mathcal{A}_{i, 2}^{(1)} \mathcal{A}_{i, 0}^{\star(0)}\left(4 A_{1}^{q}\right)+\mathcal{A}_{i, 1}^{(1)} \mathcal{A}_{i,-1}^{\star(1)}+\mathcal{A}_{i, 1}^{(1)} \mathcal{A}_{i, 0}^{\star(0)}\left(-2 f_{1}^{q}-4 B_{1}^{q}\right)+\mathcal{A}_{i,-1}^{(1)} \mathcal{A}_{i, 1}^{\star(1)} \\
& +\mathcal{A}_{i,-2}^{(1)} \mathcal{A}_{i, 2}^{\star(1)}+\mathcal{A}_{i, 0}^{(1)} \mathcal{A}_{i, 0}^{\star(0)}\left(2 \overline{\mathcal{G}}_{1}^{q,(1)}\right)+\mathcal{A}_{i, 0}^{(0)} \mathcal{A}_{i, 0}^{\star(2)}+\mathcal{A}_{i, 0}^{(0)} \mathcal{A}_{i, 2}^{\star(1)}\left(4 A_{1}^{q}\right) \\
& \left.+\mathcal{A}_{i, 0}^{(0)} \mathcal{A}_{i, 1}^{\star(1)}\left(-2 f_{1}^{q}-4 B_{1}^{q}\right)+\mathcal{A}_{i, 0}^{(0)} \mathcal{A}_{i, 0}^{\star(1)}\left(2 \overline{\mathcal{G}}_{1}^{q,(1)}\right)\right\} \\
& +\mathcal{D}_{0}\left\{\left|\mathcal{A}_{i, 0}^{(0)}\right|^{2}\left(-2 f_{2}^{q}-4 f_{1}^{q} \overline{\mathcal{G}}_{1}^{q,(1)}-4 \beta_{0} \overline{\mathcal{G}}_{1}^{q,(1)}+16 \zeta_{3}\left(A_{1}^{q}\right)^{2}+8 \zeta_{2} A_{1}^{q} f_{1}^{q}\right)\right. \\
& \left.+\mathcal{A}_{i, 0}^{(1)} \mathcal{A}_{i, 0}^{\star(0)}\left(-2 f_{1}^{q}\right)+\mathcal{A}_{i, 0}^{(0)} \mathcal{A}_{i, 0}^{\star(1)}\left(-2 f_{1}^{q}\right)\right\} \\
& +\mathcal{D}_{1}\left\{\left|\mathcal{A}_{i, 0}^{(0)}\right|^{2}\left(4\left(f_{1}^{q}\right)^{2}+4 A_{2}^{q}+8 A_{1}^{q} \overline{\mathcal{G}}_{1}^{q,(1)}+4 \beta_{0} f_{1}^{q}-16 \zeta_{2}\left(A_{1}^{q}\right)^{2}\right)\right. \\
& \left.+\mathcal{A}_{i, 0}^{(1)} \mathcal{A}_{i, 0}^{\star(0)}\left(4 A_{1}^{q}\right)+\mathcal{A}_{i, 0}^{(0)} \mathcal{A}_{i, 0}^{\star(1)}\left(4 A_{1}^{q}\right)\right\} \\
& +\mathcal{D}_{2}\left|\mathcal{A}_{i, 0}^{(0)}\right|^{2}\left\{-12 A_{1}^{q} f_{1}^{q}-4 \beta_{0} A_{1}^{q}\right\}+\mathcal{D}_{3}\left|\mathcal{A}_{i, 0}^{0}\right|^{2}\left\{8\left(A_{1}^{q}\right)^{2}\right\} \\
&
\end{aligned}
$$

where $\zeta_{2}=1.64493407 \cdots, \zeta_{3}=1.20205690 \cdots$ and $\mathcal{A}_{i, k}^{(j)}$ are obtained from eq. (2.13) by defining $\mathcal{A}_{m n}=\mathcal{A} \delta_{m n}$ and expanding in powers of $\epsilon$ as

$$
\mathcal{A}_{i}^{(j)}(\epsilon)=\sum_{k=-2 j}^{\infty} \epsilon^{k} \mathcal{A}_{i, k}^{(j)} .
$$


The cusp $\left(A_{i}^{q}, i=1,2\right)$ and collinear anomalous dimensions $\left(B_{q}^{1}\right)$ are given by

$$
\begin{aligned}
& A_{1}^{q}=4 C_{F}, \\
& A_{2}^{q}=8 C_{F} C_{A}\left\{\frac{67}{18}-\zeta_{2}\right\}+8 C_{F} n_{f}\left\{-\frac{5}{9}\right\}, \\
& B_{1}^{q}=3 C_{F},
\end{aligned}
$$

and the soft anomalous dimensions are

$$
\begin{aligned}
f_{1}^{q} & =0 \\
f_{2}^{q} & =C_{A} C_{F}\left\{-\frac{22}{3} \zeta_{2}-28 \zeta_{3}+\frac{808}{27}\right\}+C_{F} n_{f} T_{F}\left\{\frac{8}{3} \zeta_{2}-\frac{224}{27}\right\} .
\end{aligned}
$$

The universal constants $\overline{\mathcal{G}}_{k}^{q,(j)}$ are given by

$$
\begin{aligned}
& \overline{\mathcal{G}}_{1}^{q,(1)}=C_{F}\left(-3 \zeta_{2}\right), \\
& \overline{\mathcal{G}}_{2}^{q,(1)}=C_{F}\left(\frac{7}{3} \zeta_{3}\right), \\
& \overline{\mathcal{G}}_{1}^{q,(2)}=C_{F} n_{f}\left(-\frac{328}{81}+\frac{70}{9} \zeta_{2}+\frac{32}{3} \zeta_{3}\right)+C_{A} C_{F}\left(\frac{2428}{81}-\frac{469}{9} \zeta_{2}+4 \zeta_{2}{ }^{2}-\frac{176}{3} \zeta_{3}\right) .
\end{aligned}
$$

Finally, defining $\bar{\Delta}_{b \bar{b}}^{\mathrm{SV}}\left(z, Q^{2}, \mu_{F}, \mu_{R}\right)$ by

$$
\begin{aligned}
\bar{\Delta}_{b \bar{b}}^{\mathrm{SV}}\left(z, Q^{2}, \mu_{F}, \mu_{R}\right)= & \frac{1}{2 s} \prod_{n=1}^{2} \int d \phi\left(q_{n}\right)(2 \pi)^{d} \delta^{d}\left(p_{1}+p_{2}-\sum_{n=1}^{2} q_{n}\right) \\
& \times \bar{\sum}\left|\mathcal{M}_{b \bar{b}}^{(0)}\right|^{2} \sum_{i=1}^{2} \Delta_{i, b \bar{b}}^{\mathrm{SV}}\left(\left\{p_{j} \cdot q_{k}\right\}, z, Q^{2}, \mu_{F}, \mu_{R}\right),
\end{aligned}
$$

we obtain $\sigma^{H H, S V}$ :

$$
\left.\sigma^{H H, \mathrm{SV}}=\int_{\tau}^{1} d x \Phi_{a_{1} a_{2}}\left(x, \mu_{F}\right) \int_{\frac{\tau}{x}}^{1} d z \bar{\Delta}_{a_{1} a_{2}}^{\mathrm{SV}}\left(z, Q^{2}, \mu_{F}, \mu_{R}\right)\right)\left.\right|_{Q^{2}=x z S} .
$$

We have used the above formula to study the numerical impact of SV part of the partonic cross section resulting from class-B diagrams up to NNLO level on the inclusive production of a pair of Higgs bosons.

\section{Phenomenology}

In this section, we present in detail the numerical impact of our analytical results obtained in the previous sections. We mainly focus on the inclusive cross section for producing a pair of Higgs bosons at the LHC with the center-of-mass energy $\sqrt{S}=14 \mathrm{TeV}$. We use MMHT2014(68cl) PDF set [87] and the corresponding $a_{s}$ through the LHAPDF-6 [88] interface at every order in perturbation theory. We use the running bottom quark mass renormalized in $\overline{M S}$ (see eq. (13) in [42]) scheme with the boundary condition $\bar{m}_{b}\left(m_{b}\right)=$ 
$4.7 \mathrm{GeV}$. Both $a_{s}\left(\mu_{R}\right)$ and $m_{b}\left(\mu_{R}\right)$ at various orders in perturbation theory are evolved using appropriate QCD $\beta$-function coefficients and quark mass anomalous dimensions. Similarly, the PDFs are evolved to factorization scale $\mu_{F}$ using the splitting functions computed to desired accuracy in the perturbation theory. We choose the Higgs boson mass $m_{h}=125 \mathrm{GeV}$ and its total decay width $\Gamma_{h}=0.001 \mathrm{GeV}$. In our analysis, we have included all the partonic channels upto NNLO level for the class-A diagrams while for the class-B, we could do this only up to NLO level, however, at NNLO level we have included SV contributions. We find that this approximation does not change our conclusion as the dominant contribution results from class-A. To illustrate this point we state some of our observations from our numerical results. We find that the LO contributions from class-A diagrams are three orders of magnitude larger than those from class-B diagrams. We also find that NLO contributions change the LO cross section by $-1.096 \%$ and at the NNLO level the change is about $-8.095 \%$. The numerical result manifests the fact that the SV contribution presented in this paper not only gets the dominant contribution from class-A but also the stability of our NNLO result for di-Higgs production from the $b \bar{b}$ annihilation channel. We find that the contribution from bottom quark annihilation processes is two orders of magnitude smaller than from the gluon fusion processes. However, former ones need to be included for the precision studies at the LHC.

Having studied the size of the corrections both at NLO and NNLO level, it is important to quantify the uncertainties resulting from the mass scales introduced in our calculations. Recall that the renormalisation of the ultra-violet and the initial state collinear divergences enforces the introduction of mass scales namely $\mu_{R}$ and $\mu_{F}$ respectively. The $\mu_{R}$ dependency shows up in the coupling constant $a_{s}\left(\mu_{R}\right)$, the mass $m_{b}\left(\mu_{R}\right)$ and in the mass factorised partonic cross sections at various orders in perturbations theory. The coupling constants are evolved using the appropriate QCD $\beta$-function coefficients and quark mass anomalous dimensions. The $\mu_{F}$ scale dependency comes from the PDFs that are evolved using splitting functions computed in the perturbation series. But the cross section, like every other physical observables, is expected to be independent of these arbitrary mass scales. This crude fact manifests the scale independency if we sum the perturbative predictions to all orders in perturbation theory. Since we have truncated the series, there is a residual scale dependency. In the following we aim to study this by varying both $\mu_{R}$ and $\mu_{F}$ scales.

In figure 5 , we show the variation of our fixed order predictions with respect to $\mu_{R}$ (on the left panel) and $\mu_{F}$ (on the right panel) for a particular choice of central scale $\mu_{0}=250 \mathrm{GeV}$. We can see that except for the small $\mu_{R}$ and $\mu_{F}$ region, which is in the region below $\mu_{R}=m_{h}$, there is an overall reduction of the scale dependency with increasing order of perturbation theory. We observe that both NLO and NNLO results attain a much faster stability against the variation of the scales than the LO cross-section. At the leading order, there are no $\mu_{R}$ or $\mu_{F}$ scale dependent logarithms that can compensate those coming from the Yukawa coupling and parton distribution functions, and hence LO has large scale dependency. However, the inclusion of higher order terms that contain logarithms of these scales provide partial cancellation at every order in perturbation theory. Hence the inclusion of NLO and NNLO pieces reduces the dependency on the scales considerably. In 


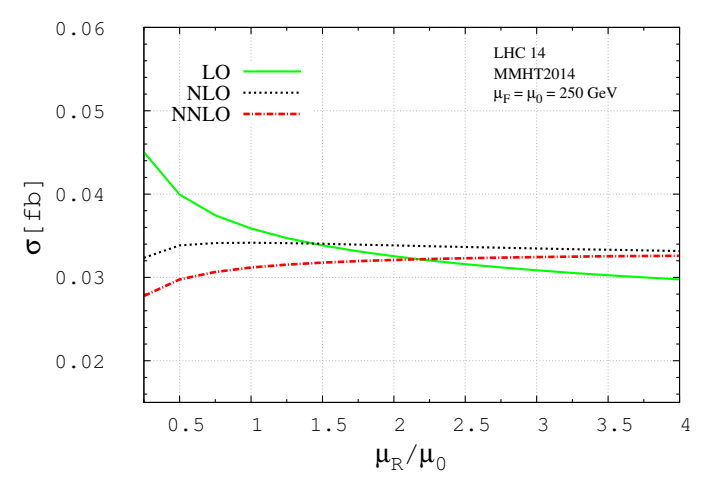

(a)

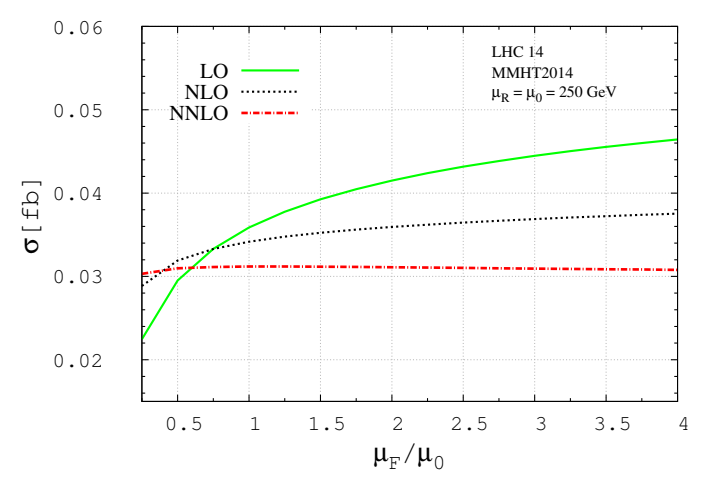

(b)

Figure 5. The total cross section for di-Higgs production in $b \bar{b}$ annihilation at various order in $a_{s}$ as a function of $\left(\mu_{R} / \mu_{0}\right)$ on left panel with $\mu_{F}=\mu_{0}$ and as a function of $\left(\mu_{F} / \mu_{0}\right)$ on right panel with $\mu_{R}=\mu_{0}$ with central scale $\mu_{0}=2 m_{h}$ and $\sqrt{s}=14 \mathrm{TeV}$.

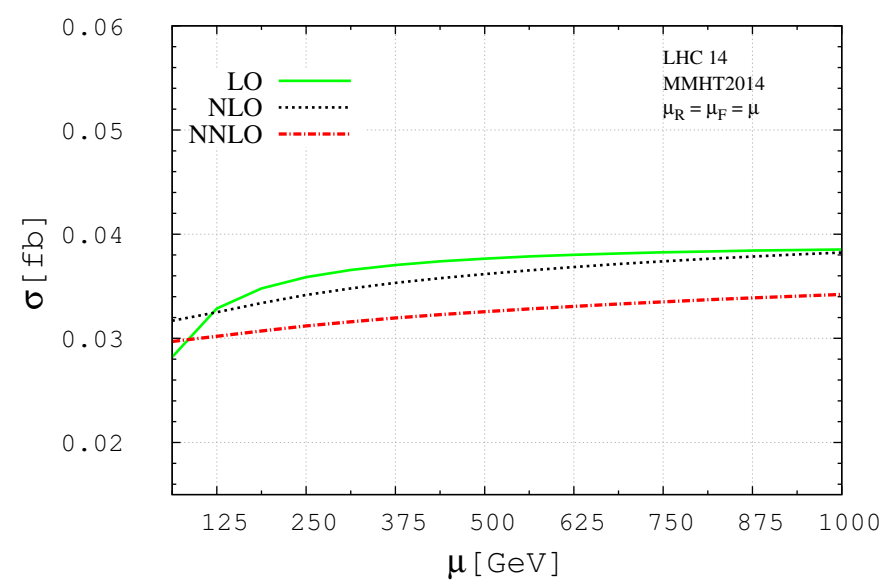

Figure 6. The total cross section for di-Higgs production in $b \bar{b}$ annihilation at various order in $a_{s}$ as a function of the mass scale $\mu$ with $\left(\mu_{F}=\mu_{R}=\mu\right)$ for $\sqrt{s}=14 \mathrm{TeV}$.

figure 6 , we have set $\mu_{R}=\mu_{F}$ and varied the cross-section with respect to a single scale $\mu$. It can be observed that LO attains stability much faster compared to the case when $\mu_{R}$ is not equal to $\mu_{F}$. This can be comprehended from figure 5 , where the LO contribution behaves exactly in an opposite way with respect to the variation of both the mass scales. So the stability in the leading order seen in figure 6 attributes to the fact that there is a significant cancellation happening between the $\mu_{R}$ and $\mu_{F}$ scale variations of the cross section. We also show the 7-point scale variation for the central scale at $m_{h}=125 \mathrm{GeV}$ in table 1 . This variation spans the entire region from $\mu_{R}, \mu_{F}=m_{h} / 2$ to $\mu_{R}, \mu_{F}=2 m_{h}$ and hence captures the uncertainty in this region. The 7 -point scale variation for a different value of central scale is also shown in table 2 . table 3 contains the $\%$ uncertainty from the scale variation at two different central scales. It can be seen that the leading order 


\begin{tabular}{|c|c|c|c|}
\hline$\left(\frac{\mu_{R}}{\kappa m_{h}}, \frac{\mu_{F}}{\kappa m_{h}}\right)$ & $\mathrm{LO}[\mathrm{fb}] \times 10^{-1}$ & $\mathrm{NLO}[\mathrm{fb}] \times 10^{-1}$ & $\mathrm{NNLO}[\mathrm{fb}] \times 10^{-1}$ \\
\hline$(2,2)$ & 0.3587 & 0.3416 & 0.3119 \\
\hline$(2,1)$ & 0.2951 & 0.3191 & 0.3098 \\
\hline$(1,2)$ & 0.3994 & 0.3384 & 0.2976 \\
\hline$(1,1)$ & 0.3286 & 0.3250 & 0.3020 \\
\hline$(1,1 / 2)$ & 0.2502 & 0.3032 & 0.3031 \\
\hline$(1 / 2,1)$ & 0.3704 & 0.3246 & 0.2879 \\
\hline$(1 / 2,1 / 2)$ & 0.2821 & 0.3169 & 0.2970 \\
\hline
\end{tabular}

Table 1. 7-point scale variation for central scale at $m_{h}=125 \mathrm{GeV}, \kappa=1$.

\begin{tabular}{|c|c|c|c|}
\hline$\left(\frac{\mu_{R}}{\kappa m_{h}}, \frac{\mu_{F}}{\kappa m_{h}}\right)$ & $\mathrm{LO}[\mathrm{fb}] \times 10^{-1}$ & $\mathrm{NLO}[\mathrm{fb}] \times 10^{-1}$ & $\mathrm{NNLO}[\mathrm{fb}] \times 10^{-1}$ \\
\hline$(2,2)$ & 0.3765 & 0.3617 & 0.3256 \\
\hline$(2,1)$ & 0.3254 & 0.3384 & 0.3210 \\
\hline$(1,2)$ & 0.4150 & 0.3594 & 0.3110 \\
\hline$(1,1)$ & 0.3587 & 0.3416 & 0.3119 \\
\hline$(1,1 / 2)$ & 0.2951 & 0.3191 & 0.3098 \\
\hline$(1 / 2,1)$ & 0.3994 & 0.3384 & 0.2976 \\
\hline$(1 / 2,1 / 2)$ & 0.3286 & 0.3250 & 0.3020 \\
\hline
\end{tabular}

Table 2. 7-point scale variation for central scale at $m_{h}=125 \mathrm{GeV}, \kappa=2$.

\begin{tabular}{|c|c|c|c|}
\hline Central Scale $(\mathrm{GeV})$ & $\mathrm{LO}[\mathrm{fb}] \times 10^{-1}$ & $\mathrm{NLO}[\mathrm{fb}] \times 10^{-1}$ & $\mathrm{NNLO}[\mathrm{fb}] \times 10^{-1}$ \\
\hline 125 & $0.3286_{-23.859 \%}^{+21.546 \%}$ & $0.3250_{-6.708 \%}^{+5.108 \%}$ & $0.3020_{-4.669 \%}^{+3.278 \%}$ \\
\hline 250 & $0.3587_{-17.731 \%}^{+15.696 \%}$ & $0.3416_{-6.587 \%}^{+5.210 \%}$ & $0.3119_{-4.585 \%}^{+4.392 \%}$ \\
\hline
\end{tabular}

Table 3. \% scale uncertainty at LO, NLO and NNLO.

cross-section has a huge scale uncertainty which implies the unreliability of the result. But the scale dependency starts to reduce when we include the higher order corrections.

\section{Conclusion}

The extraction of the trilinear coupling of the Higgs bosons provides the valuable information on the shape of the Higgs potential. One of the most important observables sensitive to this coupling is the production of a pair of Higgs bosons at the LHC. Among various partonic channels that contribute to this process, gluon fusion is the dominant one and is well studied both in effective theory as well as in the full theory. In the effective theory, top quarks are integrated out. As the precision at the hadron collider improves, it is important to incorporate other sub-dominant channels to the production mechanism. In this paper, we have considered one such channel, namely the production of a pair of Higgs bosons in the bottom quark annihilation which is sensitive to the trilinear coupling. Both LO and NLO 
QCD contributions exist in the literature and hence we have computed the NNLO QCD corrections to the inclusive cross section. There are two classes of diagrams that contribute at two-loops as well as at real emission sub processes. The vertex type of virtual diagrams that belongs to class-A is already known up to three loops and hence we evaluate only the Class-B diagrams up to two loops in perturabative QCD for our NNLO predictions. Our results are expressed in terms of classical polylogarithms of weight up to 4 . We observe that the infrared poles of the amplitudes are in agreement with the predictions by Catani. We have studied the numerical stability of the coefficient $\mathcal{C}_{2}^{(2) \text {,fin }}$ over the range of $x$ and $\cos (\theta)$ required for further phenomenological studies.

Using these results at hand, we have computed full NNLO corrections from classA diagrams and SV contributions at NNLO level from class-B diagrams to the inclusive cross section. We have shown how the NNLO results that are already available for the single Higgs boson production can be used to obtain the full NNLO contributions for the production of pair of Higgs bosons from class-A diagrams. Exploiting the universal structure of the soft contributions and the two loop amplitudes computed in this paper, we derive the SV contributions from class-B diagrams up to NNLO level. Our detailed study on the numerical impact of these results at the LHC energy demonstrates that the inclusion of higher order terms reduces the renormalization and factorization scale uncertainties making the predictions more reliable.

\section{Acknowledgments}

We sincerely thank the referee for the valuable comments and suggestions. We would like to thank Neelima Agarwal, Sophia Borowka, Goutam Das, Thomas Gehrmann, M. C. Kumar and Anurag Tripathi for several useful discussions. We thank Johannes Blümlein for carefully reading the manuscript and providing us with his valuable comments. Ajjath $\mathrm{AH}$ and Pooja Mukherjee would like to thank their parents, siblings and friends for the continuous love and support.

Open Access. This article is distributed under the terms of the Creative Commons Attribution License (CC-BY 4.0), which permits any use, distribution and reproduction in any medium, provided the original author(s) and source are credited.

\section{References}

[1] CMS collaboration, Observation of a new boson at a mass of $125 \mathrm{GeV}$ with the CMS experiment at the LHC, Phys. Lett. B 716 (2012) 30 [arXiv:1207.7235] [INSPIRE].

[2] ATLAS collaboration, Observation of a new particle in the search for the standard model Higgs boson with the ATLAS detector at the LHC, Phys. Lett. B 716 (2012) 1 [arXiv: 1207.7214] [INSPIRE].

[3] C. Englert et al., Precision measurements of Higgs couplings: implications for new physics scales, J. Phys. G 41 (2014) 113001 [arXiv:1403.7191].

[4] T. Binoth, S. Karg, N. Kauer and R. Ruckl, Multi-Higgs boson production in the Standard Model and beyond, Phys. Rev. D 74 (2006) 113008 [hep-ph/0608057] [INSPIRE]. 
[5] ATLAS collaboration, Prospects for measuring Higgs pair production in the channel $H(\rightarrow \gamma \gamma) H(\rightarrow b \bar{b})$ using the ATLAS detector at the HL-LHC, ATL-PHYS-PUB-2014-019 (2014).

[6] ATLAS collaboration, Higgs pair production in the $H(\rightarrow \tau \tau) H(\rightarrow b \bar{b})$ channel at the High-Luminosity LHC, ATL-PHYS-PUB-2015-046 (2015).

[7] CMS collaboration, Higgs pair production at the High Luminosity LHC, CMS-PAS-FTR-15-002 (Higgs pair production at the High Luminosity LHC).

[8] CMS collaboration, Updates on projections of physics reach with the upgraded CMS detector for high luminosity LHC, CMS-DP-2016-064 (2016).

[9] H.P. Nilles, Supersymmetry, supergravity and particle physics, Phys. Rept. 110 (1984) 1.

[10] E.W.N. Glover and J.J. van der Bij, Higgs boson pair production via gluon fusion, Nucl. Phys. B 309 (1988) 282 [INSPIRE].

[11] O.J.P. Eboli, G.C. Marques, S.F. Novaes and A.A. Natale, Twin Higgs boson production, Phys. Lett. B 197 (1987) 269 [inSPIRE].

[12] T. Plehn, M. Spira and P.M. Zerwas, Pair production of neutral Higgs particles in gluon-gluon collisions, Nucl. Phys. B 479 (1996) 46 [hep-ph/9603205] [INSPIRE].

[13] S. Dawson, S. Dittmaier and M. Spira, Neutral Higgs boson pair production at hadron colliders: QCD corrections, Phys. Rev. D 58 (1998) 115012 [hep-ph/9805244] [INSPIRE].

[14] A. Djouadi, W. Kilian, M. Muhlleitner and P.M. Zerwas, Testing Higgs selfcouplings at $e^{+} e^{-}$ linear colliders, Eur. Phys. J. C 10 (1999) 27 [hep-ph/9903229] [INSPIRE].

[15] A. Djouadi, W. Kilian, M. Muhlleitner and P.M. Zerwas, Production of neutral Higgs boson pairs at LHC, Eur. Phys. J. C 10 (1999) 45 [hep-ph/9904287] [InSPIRE].

[16] M.M. Mühlleitner, Higgs particles in the standard model and supersymmetric theories, Ph.D. thesis, Hamburg University, Hamburg, Germany (2000), hep-ph/0008127.

[17] J. Grigo, J. Hoff, K. Melnikov and M. Steinhauser, On the Higgs boson pair production at the LHC, Nucl. Phys. B 875 (2013) 1 [arXiv:1305.7340] [INSPIRE].

[18] R. Frederix et al., Higgs pair production at the LHC with NLO and parton-shower effects, Phys. Lett. B 732 (2014) 142 [arXiv:1401.7340] [INSPIRE].

[19] F. Maltoni, E. Vryonidou and M. Zaro, Top-quark mass effects in double and triple Higgs production in gluon-gluon fusion at NLO, JHEP 11 (2014) 079 [arXiv: 1408.6542] [INSPIRE].

[20] G. Degrassi, P.P. Giardino and R. Gröber, On the two-loop virtual QCD corrections to Higgs boson pair production in the Standard Model, Eur. Phys. J. C 76 (2016) 411 [arXiv: 1603.00385] [INSPIRE].

[21] R. Gröber, A. Maier and T. Rauh, Reconstruction of top-quark mass effects in Higgs pair production and other gluon-fusion processes, JHEP 03 (2018) 020 [arXiv:1709.07799] [INSPIRE].

[22] R. Bonciani, G. Degrassi, P.P. Giardino and R. Gröber, Analytical method for next-to-leading-order QCD corrections to double-Higgs production, Phys. Rev. Lett. 121 (2018) 162003 [arXiv: 1806.11564] [INSPIRE].

[23] S. Borowka et al., Higgs boson pair production in gluon fusion at next-to-leading order with full top-quark mass dependence, Phys. Rev. Lett. 117 (2016) 012001 [arXiv:1604.06447] [INSPIRE]. 
[24] S. Borowka et al., Full top quark mass dependence in Higgs boson pair production at NLO, JHEP 10 (2016) 107 [arXiv: 1608.04798].

[25] D. de Florian and J. Mazzitelli, Higgs boson pair production at next-to-next-to-leading order in QCD, Phys. Rev. Lett. 111 (2013) 201801 [arXiv:1309.6594].

[26] D. de Florian and J. Mazzitelli, Two-loop virtual corrections to Higgs pair production, Phys. Lett. B 724 (2013) 306 [arXiv: 1305.5206] [INSPIRE].

[27] J. Grigo, J. Hoff and M. Steinhauser, Higgs boson pair production: top quark mass effects at NLO and NNLO, Nucl. Phys. B 900 (2015) 412 [arXiv:1508.00909] [inSPIRE].

[28] Q. Li, Q.-S. Yan and X. Zhao, Higgs pair production: improved description by matrix element matching, Phys. Rev. D 89 (2014) 033015 [arXiv: 1312.3830] [INSPIRE].

[29] P. Maierhöfer and A. Papaefstathiou, Higgs Boson pair production merged to one jet, JHEP 03 (2014) 126 [arXiv: 1401.0007] [INSPIRE].

[30] D.Y. Shao, C.S. Li, H.T. Li and J. Wang, Threshold resummation effects in Higgs boson pair production at the LHC, JHEP 07 (2013) 169 [arXiv:1301.1245] [INSPIRE].

[31] D. de Florian and J. Mazzitelli, Higgs pair production at next-to-next-to-leading logarithmic accuracy at the LHC, JHEP 09 (2015) 053 [arXiv: 1505.07122] [INSPIRE].

[32] M. Grazzini et al., Higgs boson pair production at NNLO with top quark mass effects, JHEP 05 (2018) 059 [arXiv: 1803.02463] [INSPIRE].

[33] P. Banerjee et al., Two-loop massless QCD corrections to the $g+g \rightarrow H+H$ four-point amplitude, JHEP 11 (2018) 130 [arXiv:1809.05388] [INSPIRE].

[34] M.A.G. Aivazis, J.C. Collins, F.I. Olness and W.K. Tung, Leptoproduction of heavy quarks. 2. A unified $Q C D$ formulation of charged and neutral current processes from fixed target to collider energies, Phys. Rev. D 50 (1994) 3102 [hep-ph/9312319].

[35] J.C. Collins, Hard scattering factorization with heavy quarks: a general treatment, Phys. Rev. D 58 (1998) 094002 [hep-ph/9806259] [INSPIRE].

[36] M. Krämer, F.I. Olness and D.E. Soper, Treatment of heavy quarks in deeply inelastic scattering, Phys. Rev. D 62 (2000) 096007 [hep-ph/0003035] [INSPIRE].

[37] D.A. Dicus and S. Willenbrock, Higgs boson production from heavy quark fusion, Phys. Rev. D 39 (1989) 751 [INSPIRE].

[38] D. Dicus, T. Stelzer, Z. Sullivan and S. Willenbrock, Higgs boson production in association with bottom quarks at next-to-leading order, Phys. Rev. D 59 (1999) 094016 [hep-ph/9811492] [INSPIRE].

[39] F. Maltoni, Z. Sullivan and S. Willenbrock, Higgs-boson production via bottom-quark fusion, Phys. Rev. D 67 (2003) 093005 [hep-ph/0301033] [InSPIRE].

[40] F.I. Olness and W.-K. Tung, When is a heavy quark not a parton? Charged Higgs production and heavy quark mass effects in the QCD based parton model, Nucl. Phys. B 308 (1988) 813 [INSPIRE].

[41] J.F. Gunion et al., Neutral and charged Higgs detection: heavy quark fusion, top quark mass dependence and rare decays, Nucl. Phys. B 294 (1987) 621 [inSPIRE].

[42] R.V. Harlander and W.B. Kilgore, Higgs boson production in bottom quark fusion at next-to-next-to leading order, Phys. Rev. D 68 (2003) 013001 [hep-ph/0304035] [INSPIRE]. 
[43] T. Ahmed, M. Mahakhud, P. Mathews, N. Rana and V. Ravindran, Two-loop QCD corrections to Higgs $\rightarrow b+\bar{b}+g$ amplitude, JHEP 08 (2014) 075 [arXiv:1405.2324] [INSPIRE].

[44] T. Gehrmann and D. Kara, The Hbb form factor to three loops in QCD, JHEP 09 (2014) 174 [arXiv: 1407.8114] [INSPIRE].

[45] T. Ahmed, N. Rana and V. Ravindran, Higgs boson production through $b \bar{b}$ annihilation at threshold in $N^{3} L O Q C D$, JHEP 10 (2014) 139 [arXiv:1408.0787].

[46] T. Ahmed, M.K. Mandal, N. Rana and V. Ravindran, Higgs rapidity distribution in $b \bar{b}$ annihilation at threshold in $N^{3} L O Q C D$, JHEP 02 (2015) 131 [arXiv:1411.5301] [INSPIRE].

[47] M. Buza, Y. Matiounine, J. Smith and W.L. van Neerven, Charm electroproduction viewed in the variable flavor number scheme versus fixed order perturbation theory, Eur. Phys. J. C 1 (1998) 301 [hep-ph/9612398] [INSPIRE].

[48] I. Bierenbaum, J. Blumlein and S. Klein, Mellin moments of the $O\left(\alpha_{s}^{3}\right)$ heavy flavor contributions to unpolarized deep-inelastic scattering at $Q^{2} \gg m^{2}$ and anomalous dimensions, Nucl. Phys. B 820 (2009) 417 [arXiv:0904.3563].

[49] J. Ablinger et al., Three loop massive operator matrix elements and asymptotic Wilson coefficients with two different masses, Nucl. Phys. B 921 (2017) 585 [arXiv:1705.07030] [INSPIRE].

[50] J. Blümlein, A. De Freitas, C. Schneider and K. Schönwald, The variable flavor number scheme at next-to-leading order, Phys. Lett. B 782 (2018) 362 [arXiv:1804.03129] [INSPIRE].

[51] S. Dawson, C. Kao, Y. Wang and P. Williams, QCD corrections to Higgs pair production in bottom quark fusion, Phys. Rev. D 75 (2007) 013007 [hep-ph/0610284] [INSPIRE].

[52] S. Dawson, C. Kao and Y. Wang, SUSY QCD corrections to Higgs pair production from bottom quark fusion, Phys. Rev. D 77 (2008) 113005 [arXiv:0710.4331] [INSPIRE].

[53] H.-S. Hou et al., Pair production of charged Higgs bosons from bottom-quark fusion, Phys. Rev. D 71 (2005) 075014 [hep-ph/0502214] [INSPIRE].

[54] J.-J. Liu et al., Higgs boson pair production in the little Higgs model at hadron collider, Phys. Rev. D 70 (2004) 015001 [hep-ph/0404171] [INSPIRE].

[55] S. Catani, The singular behavior of QCD amplitudes at two loop order, Phys. Lett. B 427 (1998) 161 [hep-ph/9802439] [INSPIRE].

[56] V. Ravindran, Higher-order threshold effects to inclusive processes in QCD, Nucl. Phys. B 752 (2006) 173 [hep-ph/0603041] [INSPIRE].

[57] T. Gehrmann, L. Tancredi and E. Weihs, Two-loop master integrals for $q \bar{q} \rightarrow V V$ : the planar topologies, JHEP 08 (2013) 070 [arXiv: 1306.6344] [INSPIRE].

[58] P. Nogueira, Automatic Feynman graph generation, J. Comput. Phys. 105 (1993) 279.

[59] J.A.M. Vermaseren, New features of FORM, math-ph/0010025 [INSPIRE].

[60] A. von Manteuffel and C. Studerus, Reduze 2 - Distributed Feynman integral reduction, arXiv:1201.4330 [INSPIRE].

[61] F.V. Tkachov, A theorem on analytical calculability of four loop renormalization group functions, Phys. Lett. B 100 (1981) 65. 
[62] K.G. Chetyrkin and F.V. Tkachov, Integration by parts: the algorithm to calculate $\beta$-functions in 4 loops, Nucl. Phys. B 192 (1981) 159 [INSPIRE].

[63] T. Gehrmann and E. Remiddi, Differential equations for two loop four point functions, Nucl. Phys. B 580 (2000) 485 [hep-ph/9912329] [INSPIRE].

[64] R.N. Lee, LiteRed 1.4: a powerful tool for reduction of multiloop integrals, J. Phys. Conf. Ser. 523 (2014) 012059 [arXiv: 1310.1145] [INSPIRE].

[65] T. Gehrmann, A. von Manteuffel, L. Tancredi and E. Weihs, The two-loop master integrals for $q \bar{q} \rightarrow V V$, JHEP 06 (2014) 032 [arXiv: 1404.4853] [INSPIRE].

[66] D.J. Gross and F. Wilczek, Ultraviolet behavior of nonabelian gauge theories, Phys. Rev. Lett. 30 (1973) 1343 [INSPIRE].

[67] H.D. Politzer, Reliable perturbative results for strong interactions?, Phys. Rev. Lett. 30 (1973) 1346 [INSPIRE].

[68] W.E. Caswell, Asymptotic behavior of nonabelian gauge theories to two loop order, Phys. Rev. Lett. 33 (1974) 244 [INSPIRE].

[69] D.R.T. Jones, Two loop diagrams in Yang-Mills theory, Nucl. Phys. B 75 (1974) 531 [INSPIRE].

[70] E. Egorian and O.V. Tarasov, Two loop renormalization of the QCD in an arbitrary gauge, Teor. Mat. Fiz. 41 (1979) 26 [INSPIRE].

[71] T. Kinoshita, Mass singularities of Feynman amplitudes, J. Math. Phys. 3 (1962) 650 [INSPIRE].

[72] T.D. Lee and M. Nauenberg, Degenerate systems and mass singularities, Phys. Rev. 133 (1964) B1549 [INSPIRE].

[73] J.C. Collins, D.E. Soper and G.F. Sterman, Factorization for short distance hadron-hadron scattering, Nucl. Phys. B 261 (1985) 104 [INSPIRE].

[74] N. Kidonakis, G. Oderda and G.F. Sterman, Evolution of color exchange in QCD hard scattering, Nucl. Phys. B 531 (1998) 365 [hep-ph/9803241] [INSPIRE].

[75] A. Sen, Asymptotic behavior of the wide angle on-shell quark scattering amplitudes in nonabelian gauge theories, Phys. Rev. D 28 (1983) 860 [INSPIRE].

[76] L.W. Garland et al., The two loop QCD matrix element for $e^{+} e^{-} \rightarrow 3$ jets, Nucl. Phys. B 627 (2002) 107 [hep-ph/0112081] [inSPIRE].

[77] C. Anastasiou, E.W.N. Glover, C. Oleari and M.E. Tejeda-Yeomans, Two loop QCD corrections to massless quark gluon scattering, Nucl. Phys. B 605 (2001) 486 [hep-ph/0101304] [INSPIRE].

[78] E.W.N. Glover, C. Oleari and M.E. Tejeda-Yeomans, Two loop QCD corrections to gluon-gluon scattering, Nucl. Phys. B 605 (2001) 467 [hep-ph/0102201] [INSPIRE].

[79] Z. Bern, A. De Freitas and L.J. Dixon, Two loop helicity amplitudes for gluon-gluon scattering in QCD and supersymmetric Yang-Mills theory, JHEP 03 (2002) 018 [hep-ph/0201161] [INSPIRE].

[80] Z. Bern, L.J. Dixon and D.A. Kosower, Two-loop $g \rightarrow$ gg splitting amplitudes in QCD, JHEP 08 (2004) 012 [hep-ph/0404293] [INSPIRE]. 
[81] G.F. Sterman and M.E. Tejeda-Yeomans, Multiloop amplitudes and resummation, Phys. Lett. B 552 (2003) 48 [hep-ph/0210130] [INSPIRE].

[82] S.M. Aybat, L.J. Dixon and G.F. Sterman, The two-loop anomalous dimension matrix for soft gluon exchange, Phys. Rev. Lett. 97 (2006) 072001 [hep-ph/0606254] [INSPIRE].

[83] S.M. Aybat, L.J. Dixon and G.F. Sterman, The two-loop soft anomalous dimension matrix and resummation at next-to-next-to leading pole, Phys. Rev. D 74 (2006) 074004 [hep-ph/0607309] [INSPIRE].

[84] T. Becher and M. Neubert, Infrared singularities of scattering amplitudes in perturbative QCD, Phys. Rev. Lett. 102 (2009) 162001 [Erratum ibid. 111 (2013) 199905] [arXiv: 0901.0722] [INSPIRE].

[85] E. Gardi and L. Magnea, Factorization constraints for soft anomalous dimensions in QCD scattering amplitudes, JHEP 03 (2009) 079 [arXiv:0901.1091] [INSPIRE].

[86] B.W. Harris and J.F. Owens, The two cutoff phase space slicing method, Phys. Rev. D 65 (2002) 094032 [hep-ph/0102128] [INSPIRE].

[87] L.A. Harland-Lang, A.D. Martin, P. Motylinski and R.S. Thorne, Parton distributions in the LHC era: MMHT 2014 PDFs, Eur. Phys. J. C 75 (2015) 204 [arXiv: 1412.3989] [InSPIRE].

[88] A. Buckley et al., LHAPDF6: parton density access in the LHC precision era, Eur. Phys. J. C 75 (2015) 132 [arXiv:1412.7420] [INSPIRE]. 